\title{
Risk Assessment of a Wind Turbine Using an AHP-MABAC Approach with Grey System Theory: A Case Study of Morocco
}

\author{
Rim Bakhat $\mathbb{D}^{1}$ and Mohammed Rajaa ${ }^{2}$ \\ ${ }^{1}$ Management Logistics and Applied Management Department, School of Economics Sciences, University of Abdel Malek Essaidi, \\ Tangier City 90060, Morocco \\ ${ }^{2}$ Management Logistics and Applied Management Department, School of Economics Sciences, University of Abdel Malek Essaidi, \\ Tetouan City 93000, Morocco \\ Correspondence should be addressed to Rim Bakhat; rbakhat@uae.ac.ma
}

Received 11 February 2020; Revised 17 June 2020; Accepted 8 July 2020; Published 13 August 2020

Academic Editor: Sajad Azizi

Copyright ( 2020 Rim Bakhat and Mohammed Rajaa. This is an open access article distributed under the Creative Commons Attribution License, which permits unrestricted use, distribution, and reproduction in any medium, provided the original work is properly cited.

\begin{abstract}
Clean energy has become a growing concern, and many organizations pay attention to environmental protection and energy production as well. In the last few decades, the wind turbine has become the core of clean energy production and has advanced in generating electricity from $40 \mathrm{~kW}$ to $5 \mathrm{~mW}$. However, the new design of the wind turbine causes several potential failures which frequently lead to the inability to accomplish the operational requirements intended to meet the customers' expectations. As a solution to this problem, the present paper proposes a novel systematic approach that combines Multicriteria Decision-Making (MCDM) techniques and Failure Mode Effects and Criticality Analysis (FMECA) tool to reveal the fatal failures and optimize the maintenance actions. To further develop the preceding framework, this work will not only rely on the three risk factors that are involved in the traditional Risk Priority Numbers (RPN) approach but also will consider the economic aspect of the system. In the proposed approach, the grey Analytic Hierarchy Process (AHP) method is applied in the first place to calculate the weights of the four risk factors criteria. Second, the grey Multiattribute Border Approximation area Comparison (MABAC) technique is applied to rank the failure modes and their criticality on the whole system. The proposed model is verified within an organization of renewable energy production in Morocco. Furthermore, the results of the comparative and the sensitivity analysis affirm that the proposed research framework is adequate for enhancing other complex systems design, especially in a developing world where funds and resources are scarce.
\end{abstract}

\section{Introduction}

In the recent decades, maintenance has become a widely used concept and an indispensable research issue for both management and engineering disciplines. The exploitation of reliability and maintainability concepts has significantly increased to ameliorate the preventive measures and the capability of the system in responding to emergencies. Technically, most systems embody as an amalgamation of many other interconnected components which must all function together to accomplish the operational requirements [1].

As a whole, several organizations seek to determine the major causes of the failures, measure their risks, and adopt adequate practical measures to alleviate and control them. According to IEC 31010: 2019, numerous risk assessment procedures could be used including Root Cause Analysis (RCA), Fault Tree Analysis (FTA), Event Tree Analysis (ETA), Hazard and Operability Analysis (HAZOP), and Failure Mode and Effect Analysis (FMEA) [2].

Failure Mode and Effect Analysis (FMEA) is a reliability analysis tool and a design "bottom-up" approach devoted to examine the failure modes and determine their effects on the overall system functioning [3]. However, it has been applied in various industrial fields, such as the renewable energy [4-6], nuclear [7], automotive [8], and manufacturing [9]. The latter also comprises a Criticality Analysis (CA) which is a qualitative analysis technique employed to measure the 
probability of the failure modes of a system [10]. The Criticality Analysis (CA) relies on the development of the Risk Priority Number (RPN) approach attained through the multiplication of the three main risk factors: "severity (S), occurrence (O), and detection (D)" [11].

Clean energy, notably wind power, presents a current debate and that goes back to the increasing awareness of countries toward environmental protection. According to the U.S Department of Commerce, the sustainable manufacturing is "the creation of manufactured products that use processes that minimize negative environmental impacts, conserve energy and natural resources, are safe for employees, communities, and consumers, and are economically sound" [12]. Until now, the worldwide mounted numerous wind turbines with a capacity of $21 \%$ per year. At the beginning of 2020, the wind power generation will have become the pillar of clean energy and will have exceeded $15 \%$ of the global electricity production [13]. However, the traditional risk factors of the RPN approach have been criticized due to the following motives [14-17]:

(i) traditional RPN approach takes into account only three risk factors, abandoning other essential "economic aspects"

(ii) The multiplicity of the risk factors for measuring the failures risk evaluation is questionable and variant to the critical factors deviations

(iii) Multiplication operation of the risk factors is unable to detect the inherent risk in each failure mode, and that implies the lack in the mathematical foundation

(iv) Traditional attributes of the RPN approach lead to imprecise results due more to the fuzzy data collected from experts and decision-makers through the process of the risk evaluation

(v) traditional RPN approach cannot correctly measure the weights of the failure modes

(vi) The risk factors are evaluated by cross-functional decision-makers using linguistic variables which are translated into crisp numbers ranging from 1 to 10 , neglecting the ingrained ambiguity

A need remains for developing a simplified methodical risk assessment approach that serves to identify the potential failure modes and mitigate their effects on the whole system. As an answer to this challenge, this paper proposes a methodology that combines multicriteria decision-making (MCDM) methods, namely, AHP-MABAC and FMECA, to control fatal failure modes. The proposed framework is supported by the use of the Grey Systems Theory (GST) to handle the fuzziness and the ambiguity of the decisionmakers' preference judgements [18]. The main contribution of this paper is to take into consideration the economic aspect (expected costs) of the system in the RPN approach and reduce the risk factors integers to a (1-10) scale in order to decrease the risk in the assessment process [19]. Furthermore, the wind turbine has been designated, as a complex system with hierarchical levels, to verify the proposed grey model. Finally, a comparative study is performed to verify the feasibility of the proposed model, and a sensitivity analysis is carried out to check the criteria weights variation impact on the ranking results.

The remainder of the present paper is structured as follows: a literature review of relevant existing studies is presented in Section 2. In Section 3, the flowchart and research framework are provided. In Section 4, the grey AHP and grey MABAC models are developed. The application of the proposed framework in a real-world case study is furnished in Section 5. The comparative analysis and the consequences of the sensitivity analysis are outlined in Section 6. Finally, Section 7 highlights the closing remarks, study contributions, conclusions, and recommendation for future researches.

\section{Literature Review}

2.1. Risk Factors in FMECA. Nowadays, the FMECA is used in several "risk-based" industries including "engineering, automotive, medical, and chemical," [20]. This approach was initially introduced by the U.S. Military and it implies an inductive "bottom-up" risk analysis [10]. The FMECA is defined as "an analysis tool that identifies all the ways a particular component can fail, what its effects would be at the subsystem level and ultimately on the system, and what the criticality is," [21]. On the other hand, Lee defined the FMEA as "a procedure for analysis of potential failure modes within a system using the classification by the severity or evaluation of the failure's effect upon the system, where the failure modes refer categories of detailed failures according to the mechanism under a certain circumstance of the whole system" [22]. The Criticality Analysis (CA) is as an extension of the FMEA which allows analysts to quantitatively rank the potential failure modes regarding their criticality on the whole system [23]. The latter could be performed quantitatively or qualitatively and also achieved with or without available data [24]. Braglia [25] acknowledged that the FMECA is an MCDM issue and highlighted four risk factors, namely, severity, detection, occurrence, and estimated costs. Therefore, the MCDM methodology is relevant to prioritize the failure modes and eliminate the inherent risks in the traditional FMECA [26].

\subsection{Recent Applications of the AHP and MABAC Method.} In general, the Multicriteria Decision-making (MCDM) methodology is regarded as one of the most practical approaches in decision science and comprises a wide set of techniques that qualitatively and quantitatively answer the research questions [27]. Due to its practicability, several studies have used MCDM approaches to solve complex problems in the wind turbines [28-31]. Besides, the AHP method has been also employed to overcome difficulties in different areas such as "information technology" [32], "management" [33], "maintenance" [34], and "sustainable construction" [35]. On the other hand, the multiattribute border approximation area comparison (MABAC) is one of the most precise MCDM techniques that make the information as exact as possible by calculating the closeness 
coefficient values. However, the MABAC technique has captivated numerous researchers since its commencement, and it has been successfully applied in different research domains such as "transport and logistics" [36], "material evaluation and selection" [37], "system engineer evaluation" [38], and "position selection of wind farms" [39]. Based on the literature review, any research which has used a grey model, namely, AHP-MABAC, is yet to assess the failure modes in the wind turbine.

2.3. Grey Systems Theory (GST). Artificial Intelligence Models (AIMs) embrace numerous practical models which can be used with the help of computer systems such as Grey Systems Theory (GST), Fuzzy Set Theory (FST), Rough Set Theory (RST), Decision Tree (DT), Support Vector Machine (SVM), Bayesian Networks (BN), Association Rule (AR), Dempster-Shafer Theory (DST), and Case-Based Reasoning (CBR) [40]. The AIMs can solve different problems based on experts' knowledge and experiences. Therefore, the Grey Systems Theory is adopted to handle the uncertainty in the decision-making process through discrete information and partial data [41]. The latter is determined by grey formulas, grey numbers, and grey matrices, and it can be merged with the FMECA [42]. In this regard, grey system theory has been selected as the convenient artificial intelligence model due to its capacity to handle the fuzziness of experts' judgment and assess the failure modes $[43,44]$. As such, the advantages of the Grey Systems Theory over other models are briefly presented in Table 1.

The major benefit of adopting grey systems theory is manifested in its capability to formulate rational results employing a slight quantity of data $[45,46]$. However, the grey numbers may contain unfinished or partial information, but the interval that comprises their values is determined [18]. The Grey Systems Theory (GST) has been effectively employed in various research areas such as "the outsourcing logistics activities," "project risks management," "automotive industry," "information technology," "business process," and "insurance industry" [47-51].

2.4. Failure Modes in a Wind Turbine. Wind energy is one of the available powers in the world and could provide 2.600 TWh by 2020 which represents $12.3 \%$ of the global electricity provision, increasing to $21.8 \%$ by 2030 [52]. As wind energy exploitation expands, organizations require efficient solutions for expenditures management. In general, the operations and maintenance (O\&M) of a $750 \mathrm{~kW}$ onshore wind turbine costs approximately about $75-90 \%$ of the total investment costs $[13,53]$. The main reason behind these enormous costs is the lack of revealing the most critical failure modes in the assembly. However, two sorts of wind turbine systems are globally manufactured: horizontal axis wind turbine (HAWT) and vertical axis wind turbine (VAWT) depending on the sector requirements [54]. The most used sort of wind turbine in the world is 3-blade entities containing the essential parts as illustrated in Figure 1.
The main role of the rotor and blades is to transfer the wind power into mechanical power through the main shaft by the help of a gearbox and the generator. The gearbox helps the generator to accelerate the speed close to electricity production. Thus, the role of the main shaft is to support the wind turbine bearings. Placement of the wind turbine with the wind direction is made by the yaw system and nacelles [56]. A wind turbine is a complex system with hierarchical levels that transforms natural wind energy into electrical energy. The wind turbine system structure is hierarchical and entails numerous attached parts and components to form a united assembly to generate the electricity. Table 2 shows the essential components and parts of the wind turbine assembly that work altogether to produce electrical power. The rapport is developed, based on deep relevant literature and experts' knowledge in this area, to explain the relations among the indenture levels and the final indenture levels [54-56].

\section{Research Framework}

3.1. Projected Flowchart. The projected flowchart of failure mode evaluation for the wind turbine, based on the grey AHP-MABAC methodology, involves two essential phases which are graphically represented in Figure 2 . The research framework can assist decision-makers and managers in the term of

(i) Determining the risk factors that cause fatal failure modes in a system

(ii) Calculating the criteria weights of the failure modes

(iii) Ranking the most critical failure modes and enhance the system design

The details of the proposed research framework are explained as follows:

Phase I: define the research problem and structure the risk factors and failure modes: the identification of the risk factors and the failure modes comes after the research problem definition. The risk factors and the failure modes have been designated through a review of the relevant literature and contributions of experts working in the clean energy production field. As a result, four risk factors have been selected, namely, occurrence, severity, detection, and expected costs. Besides, 25 failure modes have been arranged as the most important parts of the wind turbine. Furthermore, a hierarchical framework containing the evaluation of the risk factors and the failure modes of the wind turbine is furnished and presented in Figure 2.

Phase II: grey AHP model: the grey AHP model is applied with the support of the grey systems theory to evaluate the risk factor weights by following the calculation steps described in Figure 2. This model reveals the weights of each risk factor and checks the consistency rate of the decision-makers judgements as well. Phase III: grey MABAC model: the obtained risk factors weights from the grey AHP model are integrated with 
TABLE 1: Core difference between the grey set theory and other mathematical models.

\begin{tabular}{lccc}
\hline Features & & Methods & \\
& Grey systems theory & Fuzzy mathematics & Probability statistics \\
\hline Study objects & Reduced data uncertainty & Rational uncertainty & Stochastic uncertainty \\
Model sets & Grey hazy sets & Fuzzy sets & Cantor sets \\
Conditions & Any distribution & Experience & Exact distribution \\
Techniques & Data analysis & Function of association & Probability distribution \\
Aim & Laws of reality & Rational formulas & Laws of statistics \\
Structures & Slight samples & Experience & Vast samples \\
\hline
\end{tabular}

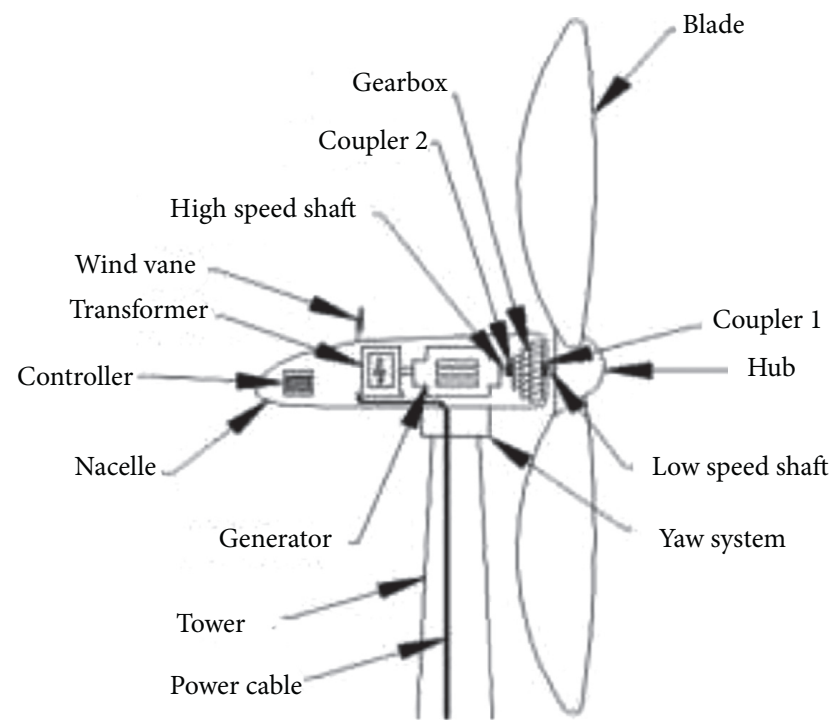

Figure 1: Onshore wind turbine hierarchical structure [55].

the grey MABAC model to select the most critical failure modes in the wind turbine. The initial decision matrix is constructed with the support of the experts.

3.2. Proposed Framework. The main role of the proposed framework within this study is to support the decision- making process and guide managers to reveal the fatal failures in the wind turbine. Also, this framework will lead to the application of a rational approach that relies on the risk priority number (RPN). In theory, the RPN considers only three types of attributes, namely, "Severity (S), Occurrence (O), and Detection (D)." Practically, the research framework has taken into account the "expected costs (ECs)" to precisely measure the failure modes. As explained in Figure 3, the proposed RPN approach applies linguistic variables to prioritize the probability of the failure modes "occurrence," the severity of the failure modes and their critical effect "rigorousness," the opportunity of the failure mode being revealed "detection," and the expected costs of the maintenance actions "expected costs."

\section{Methodology}

4.1. Preliminaries. A grey number $\otimes N$ refers to as an interval with defined upper and lower limits and undefined distribution information for $N$ [57]. In the following equation, $\underline{N}$ and $\bar{N}$. denote the lower and upper limits of $\otimes N$, correspondingly [58]:

$$
\otimes N=[\underline{N}, \bar{N}]=\left[N^{\prime} \in N \mid \underline{N} \leq N^{\prime} \leq \bar{N}\right] .
$$

In the following equations, four main grey number mathematical operations are given [43]:

$$
\begin{aligned}
& \text { Addition: } \otimes N_{1}+\otimes N_{2}=\left[\underline{N_{1}}+\underline{N_{2}}, \overline{N_{1}}+\overline{N_{2}}\right] \text {, } \\
& \text { Subtraction: } \otimes N_{1}-\otimes N_{2}=\left[\underline{N_{1}}-\overline{N_{2}}, \overline{N_{1}}-\underline{N_{2}}\right] \text {, } \\
& \text { Division: } \otimes N_{1} \div \otimes N_{2}=\left[\underline{N_{1}}, \overline{N_{1}}\right] \times\left[\frac{1}{\overline{N_{2}}}, \frac{1}{N_{2}}\right] \text {, } \\
& \text { Multiplication: } \otimes N_{1} \times \otimes N_{2}=\left[\begin{array}{l}
\min .\left(\underline{N_{1}} \underline{N_{2}}, \underline{N_{1}} \overline{N_{2}}, \overline{N_{1}} \underline{N_{2}}, \overline{N_{1} N_{2}}\right) \\
\max .\left(\underline{N_{1}} \underline{N_{2}}, \underline{N_{1}} \overline{N_{2}}, \overline{N_{1}} \underline{N_{2}}, \overline{N_{1} N_{2}}\right)
\end{array}\right] .
\end{aligned}
$$

When it comes to a crisp number, the grey aggregation method is necessary to be applied. In the present work, a "degreying" technique is hereafter applied with the support of the translating fuzzy data into Crisp Scores (CFCS)
$[59,60]$. Thus, $\otimes N_{i j}^{r}$ denotes the grey number of a crossfunctional decision-maker, who will assess the impact of risk $i$ on a risk $j$ where $N_{i j}^{r}$ and $\overline{N_{i j}^{r}}$ represent the lower and upper grey values of the grey number $\otimes N_{i j}^{r}$, similarly [58]: 
TABLE 2: Rapport among wind turbine parts.

\begin{tabular}{|c|c|c|c|c|}
\hline Failure category & Subsystem & Code & Failure modes (components) & Failure effect and criticality \\
\hline \multirow{9}{*}{ Aerodynamic system } & \multirow{2}{*}{ Blade } & $\mathrm{FM}_{1}$ & "Gear teeth slip" & "Incapability to regulate the angle" \\
\hline & & $\mathrm{FM}_{2}$ & "Blade crack" & "System halts functioning" \\
\hline & \multirow{2}{*}{ Hub assembly } & $\mathrm{FM}_{3}^{2}$ & "Error in positioning" & "Blade detach from the hub" \\
\hline & & $\mathrm{FM}_{4}$ & "Fracture in the shell" & "Rotor breaks down" \\
\hline & \multirow[t]{2}{*}{ Tower and nacelle } & $\mathrm{FM}_{5}$. & "Fracture" & "Insecure structural integrity" \\
\hline & & $\mathrm{FM}_{6}$ & "Electrical overload" & \\
\hline & \multirow[t]{2}{*}{ Pitch system } & $\mathrm{FM}_{7}$ & "Low insulation level" & "Collapse of pitch" \\
\hline & & $\mathrm{FM}_{8}$ & "Excessive loading" & \\
\hline & Yaw system & $\mathrm{FM}_{9}$ & "Fatigue or excessive loading" & "Collapse of yaw" \\
\hline \multirow{8}{*}{ Mechanical system } & \multirow{2}{*}{ System brakes } & $\mathrm{FM}_{10}$ & "Full speed" & "Downfall of other components" \\
\hline & & $\mathrm{FM}_{11}$ & "Over heating" & "Yaw and rotor breakdown" \\
\hline & \multirow{3}{*}{ Shaft set } & $\mathrm{FM}_{12}$ & "Main shaft vibration" & "Parts joining vibration" \\
\hline & & $\mathrm{FM}_{13}$ & "Main shaft malfunction" & "Shaft breakdown" \\
\hline & & $\mathrm{FM}_{14}$ & "Fatigue cracks" & "Ruin of the system" \\
\hline & \multirow{3}{*}{ Gearbox } & $\mathrm{FM}_{15}$ & "Gearbox vibration" & "Parts connection vibration" \\
\hline & & $\mathrm{FM}_{16}$ & "Gearbox malfunction" & "Gearbox breakdown" \\
\hline & & $\mathrm{FM}_{17}$ & "Gearbox abnormal noise" & \\
\hline \multirow{8}{*}{ Electrical system } & \multirow{3}{*}{ Generator } & $\mathrm{FM}_{18}$ & "Excessive fatigue" & \multirow{4}{*}{$\begin{array}{l}\text { "Failure of the generator" } \\
\text { "Rift in shaft, rotor, bearing, and stator" } \\
\text { "Loss of stator and rotor" } \\
\text { "In capability to transmit the energy } \\
\text { to the transformer" }\end{array}$} \\
\hline & & $\mathrm{FM}_{19}$ & "Poor lubrication" & \\
\hline & & $\mathrm{FM}_{20}$ & "Overheating" & \\
\hline & \multirow{3}{*}{ Centralized lubrication system } & $\mathrm{FM}_{21}$ & "Shorting the circuit" & \\
\hline & & $\mathrm{FM}_{22}$ & "Overloading" & "Collapse of the pump" \\
\hline & & $\mathrm{FM}_{23}$ & "Shorting circuit" & "Risk on electronic components" \\
\hline & \multirow{2}{*}{ Power electrical system } & $\mathrm{FM}_{24}$ & "Fatigue" & "Failure of the power system" \\
\hline & & $\mathrm{FM}_{25}$ & "Distorting" & "Collapse of the system" \\
\hline
\end{tabular}

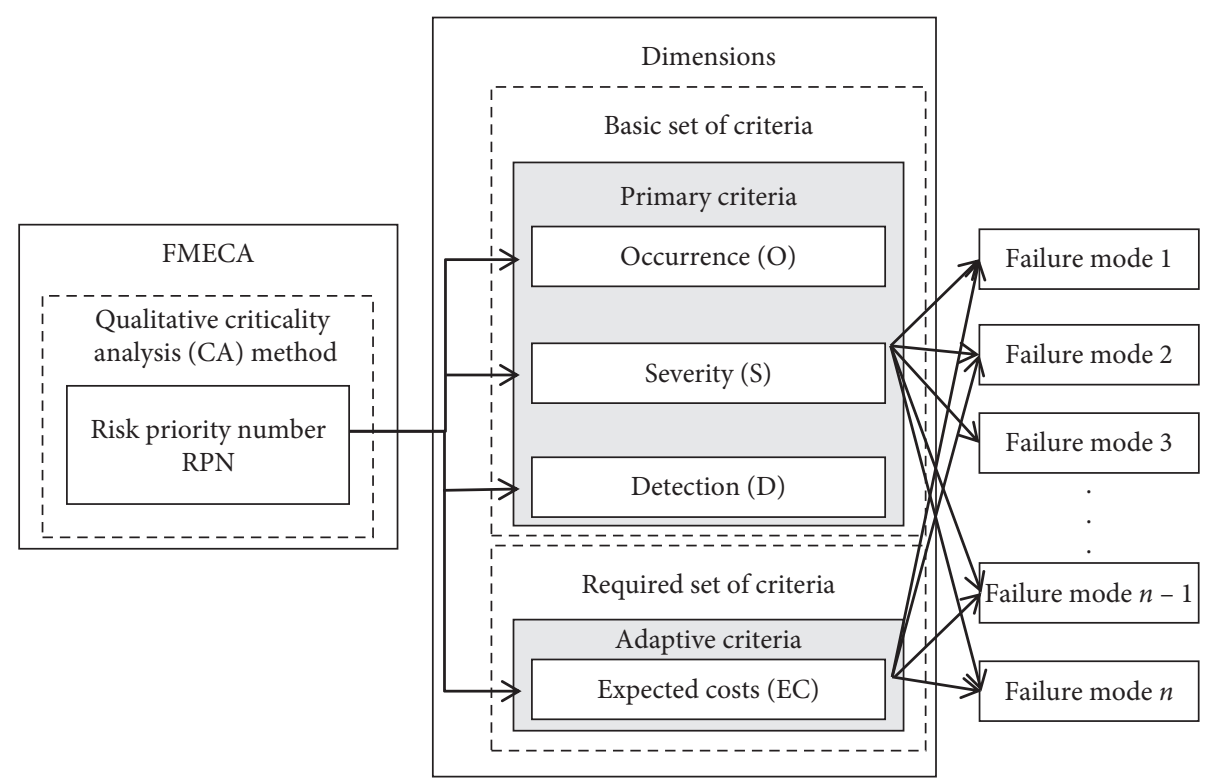

FiguRe 2: Hierarchical framework.

$$
\otimes N_{i j}^{r}=\left[\underline{N}_{i j}^{r}, \bar{N}_{i j}^{r}\right]
$$

The convertion of ambiguous data into crisp scores entails three main steps represented as follows:

Step 1: normalization:

$$
\underline{\tilde{N}}_{i j}^{r}=\frac{\left(\underline{N}_{i j}^{r}-\min _{j} \underline{N}_{i j}^{r}\right)}{\Delta_{\min }^{\max }}, \tilde{N}_{i j}^{r}=\frac{\left(\bar{N}_{i j}^{r}-\min _{j} \bar{N}_{i j}^{r}\right)}{\Delta_{\max }^{\min }},
$$

where

$$
\Delta_{\min }^{\max }=\max _{j} \bar{N}_{i j}^{r}-\min _{j} \underline{N}_{i j}^{r} .
$$




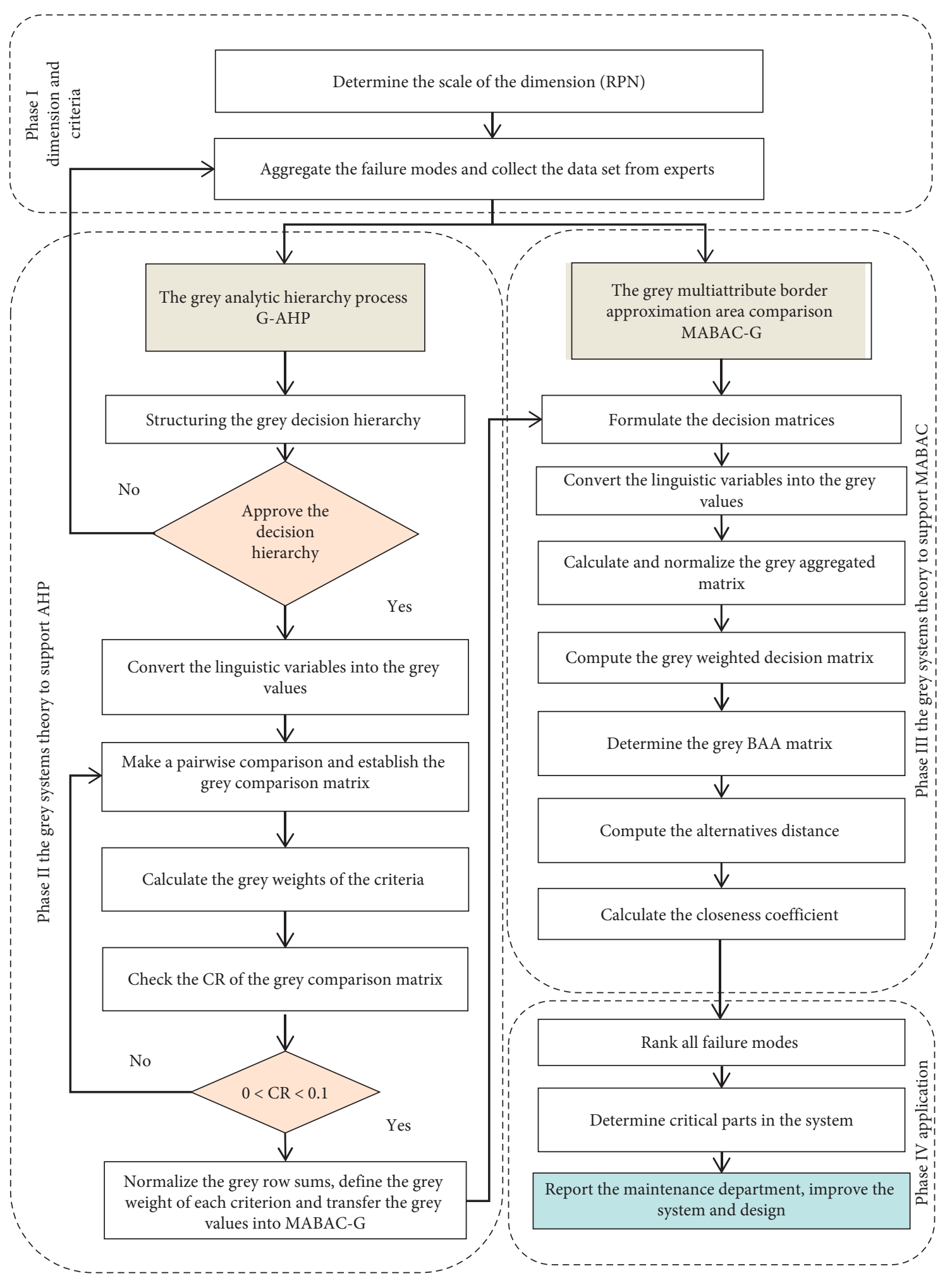

FIgURE 3: Projected flowchart. 
TABLE 3: Grey AHP model linguistic terms and grey weights [61].

\begin{tabular}{lcc}
\hline Linguistic weights & Code & Grey weights \\
\hline Absolute important & $(\mathrm{AI})$ & {$[7,9]$} \\
More important & $(\mathrm{MI})$ & {$[5,7]$} \\
Important & $(\mathrm{I})$ & {$[3,5]$} \\
Moderately important & $(\mathrm{DI})$ & {$[1,3]$} \\
Equal important & $(\mathrm{EI})$ & {$[1,1]$} \\
\hline
\end{tabular}

TABLE 4: Linguistic terms and the evaluation scale for "occurrence" $[43,49,64]$.

\begin{tabular}{lccc}
\hline Probability of failure & Code & Grey value & Probability of failure occurrence \\
\hline Extremely less & $(\mathrm{EL})$ & {$[1,2]$} & $<10^{-5}$ \\
Remote & $(\mathrm{R})$ & {$[1,3]$} & $10^{-5}$ \\
Very slight & $(\mathrm{VS})$ & {$[2,4]$} & $10^{-5}$ \\
Slight & $(\mathrm{S})$ & {$[3,5]$} & $4 \times 10^{-4}$ \\
Occasional & $(\mathrm{O})$ & {$[4,6]$} & $2 \times 10^{-3}$ \\
Moderate & $(\mathrm{M})$ & {$[5,7]$} & $1 \times 10^{-2}$ \\
Frequent & $(\mathrm{F})$ & {$[6,8]$} & $4 \times 10^{-2}$ \\
High & $(\mathrm{H})$ & {$[8,9]$} & 0.20 \\
Very high & $(\mathrm{VH})$ & {$[9,10]$} & 0.33 \\
Extremely high & $(\mathrm{EH})$ & & $\geq 0.5$ \\
\hline
\end{tabular}

TABLE 5: Linguistic terms and the evaluation scale for "severity" [43, 49, 64].

\begin{tabular}{lccc}
\hline Failure effect & Code & Grey value & Severity effect \\
\hline Very minor & $(\mathrm{VM})$ & {$[1,3]$} & Effect is not noticed' \\
Minor & $(\mathrm{MI})$ & {$[2,4]$} & Very slight effect noticed' \\
Low & $(\mathrm{L})$ & {$[3,5]$} & Slight effect causing annoyance' \\
Moderate & $(\mathrm{MO})$ & {$[4,6]$} & Moderate effected and maintenance is required' \\
Significant & $(\mathrm{S})$ & {$[5,7]$} & Significant effect and system performance is degraded' \\
Major & $(\mathrm{MA})$ & {$[6,8]$} & Major effect and system performance is affected' \\
Extreme & $(\mathrm{E})$ & {$[7,9]$} & Extreme effect, system inoperable, and safety issue' \\
Hazardous & $(\mathrm{H})$ & "Critical effect and system safety risk" \\
Very hazardous & $(\mathrm{VH})$ & {$[9,10]$} & "Higher severity ranking of a potential failure mode" \\
\hline
\end{tabular}

TABLE 6: Linguistic terms and the evaluation scale for "detection" [43, 49, 64].

\begin{tabular}{lccc}
\hline Detection & Code & Grey value & Possibility of detecting potential failures \\
\hline Almost certain & $(\mathrm{AC})$ & {$[1,2]$} & "Certain detected" \\
Very high & $(\mathrm{VH})$ & {$[1,3]$} & "Very high detection" \\
"High detection" & "Moderate detection" \\
High & $(\mathrm{H})$ & {$[2,4]$} & "Medium detection" \\
Moderately high & $(\mathrm{MH})$ & {$[3,5]$} & "Low detection" \\
Medium & $(\mathrm{M})$ & {$[4,6]$} & "Very low detection" \\
Low chance & $(\mathrm{LC})$ & {$[5,7]$} & "Remote detection" \\
Slight & $(\mathrm{S})$ & {$[6,8]$} & "Very remote detection" \\
Remote & $(\mathrm{R})$ & {$[7,9]$} & "No inspection and no chance" \\
Very remote & $(\mathrm{VR})$ & {$[9,10]$} & \\
Absolute uncertainty & $(\mathrm{AU})$ &
\end{tabular}

TABLE 7: Linguistic terms and the evaluation scale for "expected costs."

\begin{tabular}{lccc}
\hline Cost & Code & Grey value & System repair costs \\
\hline Close to original price & $(\mathrm{O})$ & {$[9,10]$} & Cost is similar to the original \\
Enormously important & $(\mathrm{EI})$ & {$[8,10]$} & Cost is enormously important' \\
Very important & $(\mathrm{VI})$ & {$[7,9]$} & Cost is very important' \\
Important & $(\mathrm{I})$ & {$[6,8]$} & Cost is important' \\
Moderately important & $(\mathrm{MI})$ & {$[5,7]$} & Cost is moderately important' \\
Moderate & $(\mathrm{M})$ & {$[4,6]$} & Cost is moderate' \\
Moderately low & $(\mathrm{ML})$ & {$[3,5]$} & Cost is quite low' \\
Soft & $(\mathrm{S})$ & {$[2,4]$} & Cost is acceptable' \\
Distant & $(\mathrm{D})$ & {$[1,3]$} & Cost is affordable' \\
Approximately no cost & $(\mathrm{N})$ & {$[1,2]$} & Almost no cost is required' \\
\hline
\end{tabular}




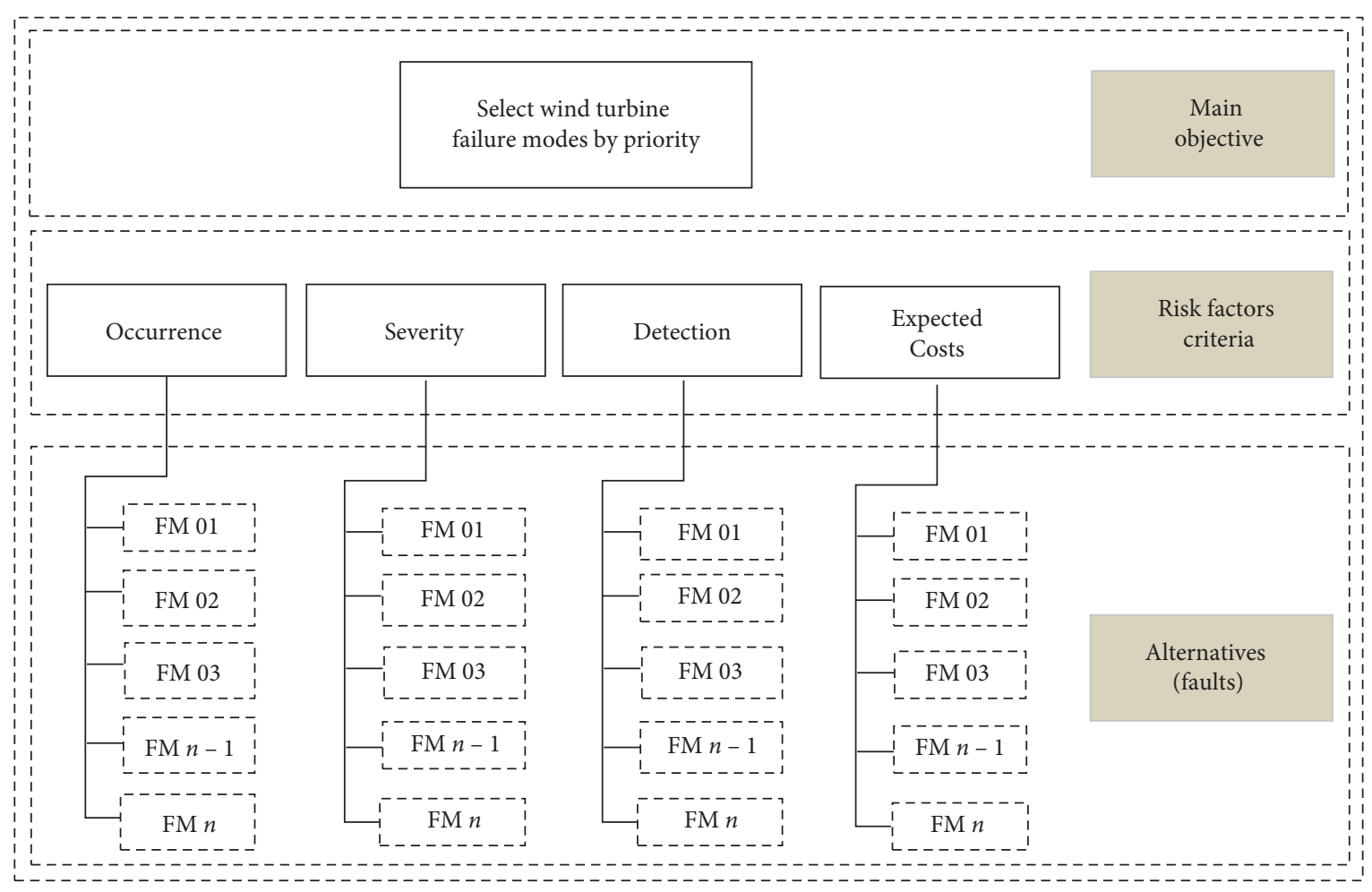

Figure 4: Hierarchical tree of the risk assessment.

TABLE 8: Grey comparison matrix.

\begin{tabular}{lcccc}
\hline Criteria & \multicolumn{4}{c}{ Criteria } \\
& $\mathrm{O}$ & $\mathrm{S}$ & $\mathrm{D}$ & $\mathrm{EC}$ \\
\hline $\mathrm{O}$ & {$[1,1]$} & {$[1,1]$} & {$[0.143,0.2]$} & {$[0.143,0.2]$} \\
$\mathrm{S}$ & {$[1,1]$} & {$[1,1]$} & {$[0.111,0.143]$} & {$[0.111,0.143]$} \\
$\mathrm{D}$ & {$[5,7]$} & {$[7,9]$} & {$[1,1]$} & {$[1,3]$} \\
$\mathrm{EC}$ & {$[5,7]$} & {$[7,9]$} & {$[0.333,1]$} & {$[1,1]$} \\
\hline
\end{tabular}

TABLe 9: Grey AHP model results.

\begin{tabular}{lcc}
\hline Criteria & \multicolumn{3}{c}{ Results } & $\otimes w_{i}$ \\
\hline $\mathrm{O}$ & {$[2.286,2.4]$} & {$[0.061,0.064]$} \\
$\mathrm{S}$ & {$[2.222,2.286]$} & {$[0.060,0.061]$} \\
$\mathrm{D}$ & {$[14,20]$} & {$[0.376,0.537]$} \\
$\mathrm{EC}$ & {$[13.333,18]$} & {$[0.358,0.483]$} \\
\hline \multicolumn{3}{c}{} \\
\hline
\end{tabular}

Step 2: computation of the total normalized crisp values:

$$
C_{i j}^{r}=\frac{\left(\tilde{N}_{i j}^{r}\left(1-\underline{\tilde{N}}_{i j}^{r}\right)+\left(\tilde{\bar{N}}_{i j}^{r} \times \tilde{\bar{N}}_{i j}^{r}\right)\right)}{\left(1-\underline{\tilde{N}}_{i j}^{r}+\tilde{\bar{N}}_{i j}^{r}\right)} .
$$

Step 3: computation of the crisp values:

$$
V_{i j}^{r}=\min _{j} \underline{N}_{i j}^{r}+C_{i j}^{r} \Delta_{\min }^{\max } .
$$

4.2. Grey AHP Model for the Risk Factors Evaluation. The grey AHP model is used to calculate the weights of risk factors. It entails three essential paces that could be explained as follows [61]:

Step 1: formulation of the grey comparison matrix: the decision-makers dispense a list of linguistic terms, as illustrated in Table 3. Then, these linguistic terms are translated into grey weights, as indicated in Table 3. Thus, the grey comparison matrix $(\otimes Q)$ is constructed as follows:

$$
\otimes Q=\left(\otimes Q_{i j}\right)_{n \times n}
$$

where

$$
\begin{aligned}
\otimes Q_{i j} & =\left[\underline{Q_{i j}}, \overline{Q_{i j}}\right], \\
Q_{i j}^{-1} & =\left[\frac{1}{\overline{Q_{i j}}}, \frac{1}{Q_{i j}}\right] .
\end{aligned}
$$

The values $Q_{i j}$ and $\overline{Q_{i j}}$ presented in equation (9) denote the minimum and the maximum values of the constructed grey comparison matrix $\otimes Q_{i j}$, respectively.

Step 2: calculation of the consistency rate: the obtained grey values are transformed into crisp values by utilizing equation (10). Afterwards, the consistency rate of the constructed grey matrix is verified with the help of equations (11) and (12) [62]. In this pace, the decision-makers calculate the consistency index (CI) and compare it to the random index (RI) for the 
TABle 10: Performance rating of the decision-makers.

\begin{tabular}{|c|c|c|c|c|}
\hline \multirow{2}{*}{ Alternatives } & \multicolumn{4}{|c|}{ Criteria } \\
\hline & $\mathrm{O}$ & S & $\mathrm{D}$ & $\mathrm{EC}$ \\
\hline $\mathrm{FM}_{1}$ & $\mathrm{R}, \mathrm{VS}, \mathrm{VS}, \mathrm{EL}, \mathrm{VS}$ & $\mathrm{VH}, \mathrm{VH}, \mathrm{H}, \mathrm{VH}, \mathrm{VH}$ & $\mathrm{MH}, \mathrm{M}, \mathrm{LC}, \mathrm{H}, \mathrm{M}$ & $\mathrm{O}, \mathrm{O}, \mathrm{EI}, \mathrm{O}, \mathrm{O}$ \\
\hline $\mathrm{FM}_{2}$ & $\mathrm{EL}, \mathrm{R}, \mathrm{EL}, \mathrm{R}, \mathrm{R}$ & VH, VH, VH, H, H & $\mathrm{VH}, \mathrm{H}, \mathrm{S}, \mathrm{VH}, \mathrm{LC}$ & M M, I, I, I \\
\hline $\mathrm{FM}_{3}$ & VS, S, R, R, R & $\mathrm{VH}, \mathrm{VH}, \mathrm{VH}, \mathrm{H}, \mathrm{VH}$ & $\mathrm{M}, \mathrm{L} \mathrm{S}, \mathrm{VH}, \mathrm{VH}$ & VI, I, EI, VI, EI \\
\hline $\mathrm{FM}_{4}$ & $\mathrm{EL}, \mathrm{R}, \mathrm{R}, \mathrm{EL}, \mathrm{EL}$ & VH, VH, H, VH, VH & $\mathrm{AC}, \mathrm{VH}, \mathrm{VH}, \mathrm{VH}, \mathrm{VH}$ & $\mathrm{O}, \mathrm{O}, \mathrm{O}, \mathrm{EI}, \mathrm{O}$ \\
\hline $\mathrm{FM}_{5}$ & $E L, R, E L, R, R$ & VM, MI, MI, MI, MI & $\mathrm{M}, \mathrm{C}, \mathrm{MH}, \mathrm{LC}, \mathrm{LC}$ & ML, ML, L, M, M \\
\hline $\mathrm{FM}_{6}$ & $\mathrm{O}, \mathrm{M}, \mathrm{M}, \mathrm{S}, \mathrm{F}$ & $\mathrm{MO}, \mathrm{S}, \mathrm{S}, \mathrm{L}, \mathrm{S}$ & $\mathrm{S}, \mathrm{R}, \mathrm{R}, \mathrm{R}$ & $\mathrm{ML}, \mathrm{M}, \mathrm{ML}, \mathrm{ML}, \mathrm{ML}$ \\
\hline $\mathrm{FM}_{7}$ & VS, S, R, O, S & S, MA, MO, E, MO & $\mathrm{M}, \mathrm{MH}, \mathrm{MH}, \mathrm{S}, \mathrm{LC}$ & $\mathrm{ML}, \mathrm{ML}, \mathrm{L}, \mathrm{D}, \mathrm{I}$ \\
\hline $\mathrm{FM}_{8}$ & VS, VS, VS, EL, VS & $\mathrm{MO}, \mathrm{S}, \mathrm{S}, \mathrm{S}, \mathrm{L}$ & $\mathrm{R}, \mathrm{VR}, \mathrm{R}, \mathrm{VR}$ & $\mathrm{M}, \mathrm{M}, \mathrm{M}, \mathrm{MI}, \mathrm{MI}$ \\
\hline $\mathrm{FM}_{9}$ & $\mathrm{M}, \mathrm{O}, \mathrm{O}, \mathrm{O}, \mathrm{O}$ & $\mathrm{VH}, \mathrm{H}, \mathrm{H}, \mathrm{MA}, \mathrm{H}$ & $\mathrm{VH}, \mathrm{H}, \mathrm{H}, \mathrm{VH}, \mathrm{AC}$ & MI, M, M, I, MI \\
\hline $\mathrm{FM}_{10}$ & R, VS, VS, EL, EL & $\mathrm{H}, \mathrm{H}, \mathrm{E}, \mathrm{MA}, \mathrm{E}$ & $\mathrm{VH}, \mathrm{H}, \mathrm{H}, \mathrm{H}, \mathrm{VH}$ & MI, MI, M, S, M \\
\hline $\mathrm{FM}_{11}$ & $\mathrm{H}, \mathrm{H}, \mathrm{F}, \mathrm{H}, \mathrm{VH}$ & $\mathrm{MO}, \mathrm{S}, \mathrm{S}, \mathrm{MO}, \mathrm{S}$ & $\mathrm{VR}, \mathrm{R}, \mathrm{R}, \mathrm{AU}, \mathrm{R}$ & ML, S, ML, ML, I \\
\hline $\mathrm{FM}_{12}$ & EL, EL, EL, EL, EL & VM, MI, MI, L, VM & VR, R, VR, R, VR & $\mathrm{N}, \mathrm{D}, \mathrm{N}, \mathrm{S}, \mathrm{D}$ \\
\hline $\mathrm{FM}_{13}$ & EL, EL, EL, R, VS & VM, MI, MI, MI, MI & $\mathrm{S}, \mathrm{LC}, \mathrm{M}, \mathrm{LC}, \mathrm{LC}$ & I, VI, VI, I, VI \\
\hline $\mathrm{FM}_{14}$ & EL, EL, R, EL, R & MI, L, L, VM, MI & LCM, M & I, I, VI, I, I \\
\hline $\mathrm{FM}_{15}$ & VS, S, S, VS, S & MI, L, VM, L, MO & $\mathrm{R}, \mathrm{S}, \mathrm{VR}, \mathrm{S}, \mathrm{M}$ & $\mathrm{N}, \mathrm{D}, \mathrm{D}, \mathrm{N}, \mathrm{D}$ \\
\hline $\mathrm{FM}_{16}$ & VS, R, VS, O, VS & $\mathrm{VH}, \mathrm{VH}, \mathrm{MI}, \mathrm{VH}, \mathrm{H}$ & $\mathrm{VH}, \mathrm{VH}, \mathrm{AC}, \mathrm{VH}, \mathrm{AC}$ & $\mathrm{O}, \mathrm{O}, \mathrm{O}, \mathrm{O}, \mathrm{EI}$ \\
\hline $\mathrm{FM}_{17}$ & $\mathrm{~S}, \mathrm{VS}, \mathrm{O}, \mathrm{VS}, \mathrm{O}$ & MI, L, VH, MO, L & $\mathrm{R}, \mathrm{VR}, \mathrm{S}, \mathrm{LC}, \mathrm{S}$ & $\mathrm{S}, \mathrm{ML}, \mathrm{D}, \mathrm{D}, \mathrm{S}$ \\
\hline $\mathrm{FM}_{18}$ & S, VS, S, VS, S & $\mathrm{VH}, \mathrm{H}, \mathrm{H}, \mathrm{L}, \mathrm{H}$ & $\mathrm{H}, \mathrm{VH}, \mathrm{VH}, \mathrm{H}, \mathrm{AU}$ & O, O, EI, EI, O \\
\hline $\mathrm{FM}_{19}$ & $\mathrm{~F}, \mathrm{VS}, \mathrm{H}, \mathrm{F}, \mathrm{H}$ & $\mathrm{VH}, \mathrm{H}, \mathrm{H}, \mathrm{H}, \mathrm{VH}$ & $\mathrm{M}, \mathrm{H}, \mathrm{R}, \mathrm{R}, \mathrm{R}, \mathrm{VR}$ & O, O, EI, VI, EI \\
\hline $\mathrm{FM}_{20}$ & $\mathrm{M}, \mathrm{F}, \mathrm{F}, \mathrm{M}, \mathrm{F}$ & $\mathrm{VH}, \mathrm{VH}, \mathrm{VH}, \mathrm{VH}, \mathrm{H}$ & $\mathrm{MH}, \mathrm{VH}, \mathrm{S}, \mathrm{AU}, \mathrm{R}$ & $\mathrm{O}, \mathrm{O}, \mathrm{O}, \mathrm{O}, \mathrm{O}$ \\
\hline $\mathrm{FM}_{21}$ & S, VS, S, S, VS & $\mathrm{VH}, \mathrm{H}, \mathrm{E}, \mathrm{H}, \mathrm{E}$ & $\mathrm{R}, \mathrm{H}, \mathrm{LC}, \mathrm{VR}, \mathrm{VR}$ & $\mathrm{N}, \mathrm{D}, \mathrm{ML}, \mathrm{D}, \mathrm{MI}$ \\
\hline $\mathrm{FM}_{22}$ & $\mathrm{~F}, \mathrm{VH}, \mathrm{S}, \mathrm{F}, \mathrm{F}$ & $\mathrm{MA}, \mathrm{E}, \mathrm{S}, \mathrm{E}, \mathrm{MO}$ & $\mathrm{S}, \mathrm{R}, \mathrm{MH}, \mathrm{LC}, \mathrm{S}$ & $\mathrm{M}, \mathrm{MI}, \mathrm{M}, \mathrm{M}, \mathrm{M}$ \\
\hline $\mathrm{FM}_{23}$ & $\mathrm{H}, \mathrm{VH}, \mathrm{VH}, \mathrm{H}, \mathrm{H}$ & $\mathrm{MO}, \mathrm{S}, \mathrm{MO}, \mathrm{S}, \mathrm{E}$ & LC, S, AU, S, M & $\mathrm{S}, \mathrm{ML}, \mathrm{D}, \mathrm{S}, \mathrm{D}$ \\
\hline $\mathrm{FM}_{24}$ & $\mathrm{H}, \mathrm{EH}, \mathrm{VH}, \mathrm{VH}, \mathrm{VH}$ & $\mathrm{S}, \mathrm{MA}, \mathrm{MO}, \mathrm{L}, \mathrm{S}$ & $\mathrm{R}, \mathrm{VR}, \mathrm{R}, \mathrm{VR}, \mathrm{R}$ & $\mathrm{D}, \mathrm{D}, \mathrm{S}, \mathrm{ML}, \mathrm{S}$ \\
\hline $\mathrm{FM}_{25}$ & $\mathrm{VH}, \mathrm{EH}, \mathrm{EH}, \mathrm{M}, \mathrm{VH}$ & $\mathrm{H}, \mathrm{H}, \mathrm{E}, \mathrm{E}, \mathrm{E}$ & $\mathrm{R}, \mathrm{VR}, \mathrm{R}, \mathrm{VR}, \mathrm{R}$ & $\mathrm{D}, \mathrm{ML}, \mathrm{D}, \mathrm{D}, \mathrm{D}, \mathrm{M}$ \\
\hline
\end{tabular}

TABle 11: Average grey decision matrix $(\widehat{T})$.

\begin{tabular}{|c|c|c|c|c|}
\hline \multirow{2}{*}{ Alternatives } & \multicolumn{4}{|c|}{ Criteria } \\
\hline & $\mathrm{O}$ & $S$ & $\mathrm{D}$ & $\mathrm{EC}$ \\
\hline Alternative $1\left(\mathrm{FM}_{1}\right)$ & {$[1,3]$} & {$[9,10]$} & {$[3,5]$} & {$[9,10]$} \\
\hline Alternative $2\left(\mathrm{FM}_{2}\right)$ & {$[1,2]$} & {$[9,10]$} & {$[1,3]$} & {$[5,7]$} \\
\hline Alternative $3\left(\mathrm{FM}_{3}\right)$ & {$[2,4]$} & {$[9,10]$} & {$[4,6]$} & {$[7,9]$} \\
\hline Alternative $4\left(\mathrm{FM}_{4}\right)$ & {$[1,2]$} & {$[9,10]$} & {$[1,2]$} & {$[9,10]$} \\
\hline Alternative $5\left(\mathrm{FM}_{5}\right)$ & {$[1,2]$} & {$[1,3]$} & {$[4,6]$} & {$[3,5]$} \\
\hline Alternative $6\left(\mathrm{FM}_{6}\right)$ & {$[4,6]$} & {$[4,6]$} & {$[6,8]$} & {$[3,5]$} \\
\hline Alternative $7\left(\mathrm{FM}_{7}\right)$ & {$[2,4]$} & {$[5,7]$} & {$[4,6]$} & {$[3,5]$} \\
\hline Alternative $8\left(\mathrm{FM}_{8}\right)$ & {$[2,4]$} & {$[4,6]$} & {$[7,9]$} & {$[4,6]$} \\
\hline Alternative $9\left(\mathrm{FM}_{9}\right)$ & {$[5,7]$} & {$[9,10]$} & {$[1,3]$} & {$[5,7]$} \\
\hline Alternative $10\left(\mathrm{FM}_{10}\right)$ & {$[1,3]$} & {$[8,10]$} & {$[1,3]$} & {$[5,7]$} \\
\hline Alternative $11\left(\mathrm{FM}_{11}\right)$ & {$[7,9]$} & {$[4,6]$} & {$[8,10]$} & {$[3,5]$} \\
\hline Alternative $12\left(\mathrm{FM}_{12}\right)$ & {$[1,2]$} & {$[1,3]$} & {$[8,10]$} & {$[1,2]$} \\
\hline Alternative $13\left(\mathrm{FM}_{13}\right)$ & {$[1,2]$} & {$[1,3]$} & {$[6,8]$} & {$[6,8]$} \\
\hline Alternative $14\left(\mathrm{FM}_{14}\right)$ & {$[1,2]$} & {$[2,4]$} & {$[5,7]$} & {$[6,8]$} \\
\hline Alternative $15\left(\mathrm{FM}_{15}\right)$ & {$[2,4]$} & {$[2,4]$} & {$[7,9]$} & {$[1,2]$} \\
\hline Alternative $16\left(\mathrm{FM}_{16}\right)$ & {$[2,4]$} & {$[9,10]$} & {$[1,3]$} & {$[9,10]$} \\
\hline Alternative $17\left(\mathrm{FM}_{17}\right)$ & {$[3,5]$} & {$[2,4]$} & {$[7,9]$} & {$[2,4]$} \\
\hline Alternative $18\left(\mathrm{FM}_{18}\right)$ & {$[3,5]$} & {$[9,10]$} & {$[2,4]$} & {$[9,10]$} \\
\hline Alternative $19\left(\mathrm{FM}_{19}\right)$ & {$[6,8]$} & {$[9,10]$} & {$[4,6]$} & {$[9,10]$} \\
\hline Alternative $20\left(\mathrm{FM}_{20}\right)$ & {$[5,7]$} & {$[9,10]$} & {$[3,5]$} & {$[9,10]$} \\
\hline Alternative $21\left(\mathrm{FM}_{21}\right)$ & {$[3,5]$} & {$[9,10]$} & {$[7,9]$} & {$[1,2]$} \\
\hline Alternative $22\left(\mathrm{FM}_{22}\right)$ & {$[6,8]$} & {$[6,8]$} & {$[6,8]$} & {$[4,6]$} \\
\hline Alternative $23\left(\mathrm{FM}_{23}\right)$ & {$[7,9]$} & {$[4,6]$} & {$[5,7]$} & {$[2,4]$} \\
\hline Alternative $24\left(\mathrm{FM}_{24}\right)$ & {$[7,9]$} & {$[5,7]$} & {$[7,9]$} & {$[1,3]$} \\
\hline Alternative $25\left(\mathrm{FM}_{25}\right)$ & {$[8,10]$} & {$[8,10]$} & {$[4,6]$} & {$[2,4]$} \\
\hline
\end{tabular}


TABLE 12: Normalized grey decision matrix $(\widehat{P})$.

\begin{tabular}{|c|c|c|c|c|}
\hline \multirow{2}{*}{ Alternatives } & \multicolumn{4}{|c|}{ Criteria } \\
\hline & $\mathrm{O}$ & $S$ & $\mathrm{D}$ & $\mathrm{EC}$ \\
\hline Alternative $1\left(\mathrm{FM}_{1}\right)$ & {$[0.0000,0.1250]$} & {$[1.0000,1.0000]$} & {$[0.7143,0.6250]$} & {$[1.0000,1.0000]$} \\
\hline Alternative $2\left(\mathrm{FM}_{2}\right)$ & {$[0.0000,0.0000]$} & {$[1.0000,1.0000]$} & {$[1.0000,0.8750]$} & {$[0.5000,0.6250]$} \\
\hline Alternative $3\left(\mathrm{FM}_{3}\right)$ & {$[0.1429,0.2500]$} & {$[1.0000,1.0000]$} & {$[0.5714,0.5000]$} & {$[0.7500,0.8750]$} \\
\hline Alternative $4\left(\mathrm{FM}_{4}\right)$ & {$[0.0000,0.0000]$} & {$[1.0000,1.0000]$} & {$[1.0000,1.0000]$} & {$[1.0000,1.0000]$} \\
\hline Alternative $5\left(\mathrm{FM}_{5}\right)$ & {$[0.0000,0.0000]$} & {$[0.0000,0.0000]$} & {$[0.5714,0.5000]$} & {$[0.2500,0.3750]$} \\
\hline Alternative $6\left(\mathrm{FM}_{6}\right)$ & {$[0.4286,0.5000]$} & {$[0.3750,0.4286]$} & {$[0.2857,0.2500]$} & {$[0.2500,0.3750]$} \\
\hline Alternative $7\left(\mathrm{FM}_{7}\right)$ & {$[0.1429,0.2500]$} & {$[0.5000,0.5714]$} & {$[0.5714,0.5000]$} & {$[0.2500,0.3750]$} \\
\hline Alternative $8\left(\mathrm{FM}_{8}\right)$ & {$[0.1429,0.2500]$} & {$[0.3750,0.4286]$} & {$[0.1419,0.1250]$} & {$[0.3750,0.5000]$} \\
\hline Alternative $9\left(\mathrm{FM}_{9}\right)$ & {$[0.5714,0.6250]$} & {$[1.0000,1.0000]$} & {$[1.0000,0.8750]$} & {$[0.5000,0.6250]$} \\
\hline Alternative $10\left(\mathrm{FM}_{10}\right)$ & {$[0.0000,0.1250]$} & {$[0.8750,1.0000]$} & {$[1.0000,0.8750]$} & {$[0.5000,0.6250]$} \\
\hline Alternative $11\left(\mathrm{FM}_{11}\right)$ & {$[0.8571,0.8750]$} & {$[0.3750,0.4286]$} & {$[0.0000,0.0000]$} & {$[0.2500,0.3750]$} \\
\hline Alternative $12\left(\mathrm{FM}_{12}\right)$ & {$[0.0000,0.0000]$} & {$[0.0000,0.0000]$} & {$[0.0000,0.0000]$} & {$[0.0000,0.0000]$} \\
\hline Alternative $13\left(\mathrm{FM}_{13}\right)$ & {$[0.0000,0.0000]$} & {$[0.0000,0.0000]$} & {$[0.2857,0.2500]$} & {$[0.6250,0.7500]$} \\
\hline Alternative $14\left(\mathrm{FM}_{14}\right)$ & {$[0.0000,0.0000]$} & {$[0.1250,0.1429]$} & {$[0.4286,0.3750]$} & {$[0.6250,0.7500]$} \\
\hline Alternative $15\left(\mathrm{FM}_{15}\right)$ & {$[0.1429,0.2500]$} & {$[0.1250,0.1429]$} & {$[0.1429,0.1250]$} & {$[0.0000,0.0000]$} \\
\hline Alternative $16\left(\mathrm{FM}_{16}\right)$ & {$[0.1429,0.2500]$} & {$[1.0000,1.0000]$} & {$[1.0000,0.8750]$} & {$[1.0000,1.0000]$} \\
\hline Alternative $17\left(\mathrm{FM}_{17}\right)$ & {$[0.2857,0.3750]$} & {$[0.1250,0.1429]$} & {$[0.1429,0.1250]$} & {$[0.1250,0.2500]$} \\
\hline Alternative $18\left(\mathrm{FM}_{18}\right)$ & {$[0.2857,0.3750]$} & {$[1.0000,1.0000]$} & {$[0.8571,0.7500]$} & {$[1.0000,1.0000]$} \\
\hline Alternative $19\left(\mathrm{FM}_{19}\right)$ & {$[0.7143,0.7500]$} & {$[1.0000,1.0000]$} & {$[0.5714,0.5000]$} & {$[1.0000,1.0000]$} \\
\hline Alternative $20\left(\mathrm{FM}_{20}\right)$ & {$[0.5714,0.6250]$} & {$[1.0000,1.0000]$} & {$[0.7143,0.6250]$} & {$[1.0000,1.0000]$} \\
\hline Alternative $21\left(\mathrm{FM}_{21}\right)$ & {$[0.2857,0.3750]$} & {$[1.0000,1.0000]$} & {$[0.1429,0.1250]$} & {$[0.0000,0.0000]$} \\
\hline Alternative $22\left(\mathrm{FM}_{22}\right)$ & {$[0.7143,0.7500]$} & {$[0.6250,0.7143]$} & {$[0.2857,0.2500]$} & {$[0.3750,0.5000]$} \\
\hline Alternative $23\left(\mathrm{FM}_{23}\right)$ & {$[0.8571,0.8750]$} & {$[0.3750,0.4286]$} & {$[0.4286,0.3750]$} & {$[0.1250,02500]$} \\
\hline Alternative $24\left(\mathrm{FM}_{24}\right)$ & {$[0.8571,0.8750]$} & {$[0.5000,0.5714]$} & {$[0.1429,0.1250]$} & {$[0.0000,0.1250]$} \\
\hline Alternative $25\left(\mathrm{FM}_{25}\right)$ & {$[1.0000,1.0000]$} & {$[0.8750,1.0000]$} & {$[0.5714,0.5000]$} & {$[0.1250,0.2500]$} \\
\hline
\end{tabular}

TABLE 13: Weighted grey decision matrix $(\widehat{G})$.

\begin{tabular}{|c|c|c|c|c|}
\hline \multirow{2}{*}{ Alternatives } & \multicolumn{4}{|c|}{ Criteria } \\
\hline & $\mathrm{O}$ & S & $\mathrm{D}$ & EC \\
\hline Alternative $1\left(\mathrm{FM}_{1}\right)$ & {$[0.0748,0.0686]$} & {$[0.1325,0.1032]$} & {$[0.8263,0.8146]$} & {$[0.7538,0.7723]$} \\
\hline Alternative $2\left(\mathrm{FM}_{2}\right)$ & {$[0.0748,0.0610]$} & {$[0.1325,0.1032]$} & {$[0.9640,0.9399]$} & {$[0.5654,0.6275]$} \\
\hline Alternative $3\left(\mathrm{FM}_{3}\right)$ & {$[0.0855,0.0762]$} & {$[0.1325,0.1032]$} & {$[0.7574,0.7519]$} & {$[0.6596,0.7240]$} \\
\hline Alternative $4\left(\mathrm{FM}_{4}\right)$ & {$[0.0748,0.0610]$} & {$[0.1325,0.1032]$} & {$[0.9640,1.0025]$} & {$[0.7538,0.7723]$} \\
\hline Alternative $5\left(\mathrm{FM}_{5}\right)$ & {$[0.0748,0.0610]$} & {$[0.0663,0.0516]$} & {$[0.7574,0.7519]$} & {$[0.4711,0.5309]$} \\
\hline Alternative $6\left(\mathrm{FM}_{6}\right)$ & {$[0.1069,0.0915]$} & {$[0.0911,0.0737]$} & {$[0.6197,0.6266]$} & {$[0.4711,0.5309]$} \\
\hline Alternative $7\left(\mathrm{FM}_{7}\right)$ & {$[0.0855,0.0762]$} & {$[0.0994,0.0811]$} & {$[0.7574,0.7519]$} & {$[0.4711,0.5309]$} \\
\hline Alternative $8\left(\mathrm{FM}_{8}\right)$ & {$[0.0855,0.0762]$} & {$[0.0911,0.0737]$} & {$[0.5508,0.5639]$} & {$[0.5183,0.5792]$} \\
\hline Alternative $9\left(\mathrm{FM}_{9}\right)$ & {$[0.1176,0.0991]$} & {$[0.1325,0.1032]$} & {$[0.9640,0.9399]$} & {$[0.5654,0.6275]$} \\
\hline Alternative $10\left(\mathrm{FM}_{10}\right)$ & {$[0.0748,0.0686]$} & {$[0.1242,0.1032]$} & {$[0.9640,0.9399]$} & {$[0.5654,0.6275]$} \\
\hline Alternative $11\left(\mathrm{FM}_{11}\right)$ & {$[0.1390,0.1143]$} & {$[0.0911,0.0737]$} & {$[0.4820,0.5013]$} & {$[0.4711,0.5309]$} \\
\hline Alternative $12\left(\mathrm{FM}_{12}\right)$ & {$[0.0748,0.0610]$} & {$[0.0663,0.0516]$} & {$[0.4820,0.5013]$} & {$[0.3769,0.3861]$} \\
\hline Alternative $13\left(\mathrm{FM}_{13}\right)$ & {$[0.0748,0.0610]$} & {$[0.0663,0.0516]$} & {$[0.6197,0.6266]$} & {$[0.6125,0.6757]$} \\
\hline Alternative $14\left(\mathrm{FM}_{14}\right)$ & {$[0.0748,0.0610]$} & {$[0.0745,0.0590]$} & {$[0.6886,0.6892]$} & {$[0.6125,0.6757]$} \\
\hline Alternative $15\left(\mathrm{FM}_{15}\right)$ & {$[0.0855,0.0762]$} & {$[0.0745,0.0590]$} & {$[0.5508,0.5639]$} & {$[0.3769,0.3861]$} \\
\hline Alternative $16\left(\mathrm{FM}_{16}\right)$ & {$[0.0855,0.0762]$} & {$[0.1325,0.1032]$} & {$[0.9640,0.9399]$} & {$[0.7538,0.7723]$} \\
\hline Alternative $17\left(\mathrm{FM}_{17}\right)$ & {$[0.0962,0.0838]$} & {$[0.0745,0.0590]$} & {$[0.5508,0.5639]$} & {$[0.4240,0.4827]$} \\
\hline Alternative $18\left(\mathrm{FM}_{18}\right)$ & {$[0.0962,0.0838]$} & {$[0.1325,0.1032]$} & {$[0.8951,0.8772]$} & {$[0.7538,0.7723]$} \\
\hline Alternative $19\left(\mathrm{FM}_{19}\right)$ & {$[0.1283,0.1067]$} & {$[0.1325,0.1032]$} & {$[0.7574,0.7519]$} & {$[0.7538,0.7723]$} \\
\hline Alternative $20\left(\mathrm{FM}_{20}\right)$ & {$[0.1176,0.0991]$} & {$[0.1325,0.1032]$} & {$[0.8263,0.8146]$} & {$[0.7538,0.7723]$} \\
\hline Alternative $21\left(\mathrm{FM}_{21}\right)$ & {$[0.0962,0.0838]$} & {$[0.1325,0.1032]$} & {$[0.5508,0.5639]$} & {$[0.3769,0.3861]$} \\
\hline Alternative $22\left(\mathrm{FM}_{22}\right)$ & {$[0.1283,0.1067]$} & {$[0.1077,0.0885]$} & {$[0.6197,0.6266]$} & {$[0.5183,0.5792]$} \\
\hline Alternative $23\left(\mathrm{FM}_{23}\right)$ & {$[0.1390,0.1143]$} & {$[0.0911,0.0737]$} & {$[0.6886,0.6892]$} & {$[0.4240,0.4827]$} \\
\hline Alternative $24\left(\mathrm{FM}_{24}\right)$ & {$[0.1390,0.1143]$} & {$[0.0994,0.0811]$} & {$[0.5508,0.5639]$} & {$[0.3769,0.4344]$} \\
\hline Alternative $25\left(\mathrm{FM}_{25}\right)$ & {$[0.1497,0.1220]$} & {$[0.1242,0.1032]$} & {$[0.7574,0.7519]$} & {$[0.4240,0.4827]$} \\
\hline
\end{tabular}


TABLE 14: Grey BAA matrix $(\widehat{B})$.

\begin{tabular}{|c|c|c|c|c|}
\hline \multirow{2}{*}{ Alternatives } & \multicolumn{4}{|c|}{ Criteria } \\
\hline & $\mathrm{O}$ & S & $\mathrm{D}$ & $\mathrm{EC}$ \\
\hline GEO & {$[0.0963,0.0819]$} & {$[0.1033,0.0821]$} & {$[0.7064,0.7088]$} & {$[0.5357,0.5815]$} \\
\hline
\end{tabular}

TABLe 15: Grey BAA matrix $(X)$.

\begin{tabular}{|c|c|c|c|c|}
\hline \multirow{2}{*}{ Alternatives } & \multicolumn{4}{|c|}{ Criteria } \\
\hline & $\mathrm{O}$ & S & $\mathrm{D}$ & EC \\
\hline Alternative $1\left(\mathrm{FM}_{1}\right)$ & {$[-0.0215,-0.0133]$} & {$[0.0292,0.0212]$} & {$[0.1198,0.1058]$} & {$[0.2181,0.1908]$} \\
\hline Alternative $2\left(\mathrm{FM}_{2}\right)$ & {$[-0.0215,-0.0209]$} & {$[0.0292,0.0212]$} & {$[0.2575,0.2311]$} & {$[0.0297,0.0460]$} \\
\hline Alternative $3\left(\mathrm{FM}_{3}\right)$ & {$[-0.0108,-0.0057]$} & {$[0.0292,0.0212]$} & {$[0.0510,0.0431]$} & {$[0.1239,0.1425]$} \\
\hline Alternative $4\left(\mathrm{FM}_{4}\right)$ & {$[-0.0215,-0.0209]$} & {$[0.0292,0.0212]$} & {$[0.2575,0.2937]$} & {$[0.2181,0.1908]$} \\
\hline Alternative $5\left(\mathrm{FM}_{5}\right)$ & {$[-0.0215,-0.0209]$} & {$[-0.0371,-0.0304]$} & {$[0.0510,0.0431]$} & {$[-0.0646,-0.0506]$} \\
\hline Alternative $6\left(\mathrm{FM}_{6}\right)$ & {$[0.0106,0.0095]$} & {$[-0.0122,-0.0083]$} & {$[-0.0867,-0.0822]$} & {$[-0.0646,-0.0506]$} \\
\hline Alternative $7\left(\mathrm{FM}_{7}\right)$ & {$[-0.0108,-0.0057]$} & {$[-0.0040,-0.0009]$} & {$[0.0510,0.0431]$} & {$[-0.0646,-0.0506]$} \\
\hline Alternative $8\left(\mathrm{FM}_{8}\right)$ & {$[-0.0108,-0.0057]$} & {$[-0.0122,-0.0083]$} & {$[-0.1556,-0.1449]$} & {$[-0.0174,0.0023]$} \\
\hline Alternative $9\left(\mathrm{FM}_{9}\right)$ & {$[0.0213,0.0172]$} & {$[0.0292,0.0212]$} & {$[0.2575,0.2311]$} & {$[0.0297,0.0460]$} \\
\hline Alternative $10\left(\mathrm{FM}_{10}\right)$ & {$[-0.0215,-0.0133]$} & {$[0.0209,0.0212]$} & {$[0.2575,0.2311]$} & {$[0.0297,0.0460]$} \\
\hline Alternative $11\left(\mathrm{FM}_{11}\right)$ & {$[0.0427,0.0324]$} & {$[-0.0122,-0.0083]$} & {$[0.2244,-0.2075]$} & {$[-0.0646,-0.0506]$} \\
\hline Alternative $12\left(\mathrm{FM}_{12}\right)$ & {$[-0.0215,-0.0209]$} & {$[-0.0371,-0.0304]$} & {$[0.2244,-0.2075]$} & {$[-0.1588,-0.1954]$} \\
\hline Alternative $13\left(\mathrm{FM}_{13}\right)$ & {$[-0.0215,-0.0209]$} & {$[-0.0371,-0.0304]$} & {$[-0.0867,-0.0822]$} & {$[0.0768,0.0942]$} \\
\hline Alternative $14\left(\mathrm{FM}_{14}\right)$ & {$[-0.0215,-0.0209]$} & {$[-0.0288,-0.0231]$} & {$[-0.0179,-0.0196]$} & {$[0.0768,0.0942]$} \\
\hline Alternative $15\left(\mathrm{FM}_{15}\right)$ & {$[-0.0108,-0.0057]$} & {$[-0.0288,-0.0231]$} & {$[-0.1556,-0.1449]$} & {$[-0.1588,-0.1954]$} \\
\hline Alternative $16\left(\mathrm{FM}_{16}\right)$ & {$[-0.0108,-0.0057]$} & {$[0.0292,0.0212]$} & {$[0.2575,0.2311]$} & {$[0.2181,0.1908]$} \\
\hline Alternative $17\left(\mathrm{FM}_{17}\right)$ & {$[-0.0001,0.0019]$} & {$[-0.0288,-0.0231]$} & {$[-0.1556,-0.1449]$} & {$[-0.1117,-0.0988]$} \\
\hline Alternative $18\left(\mathrm{FM}_{18}\right)$ & {$[-0.0001,0.0019]$} & {$[0.0292,0.0212]$} & {$[0.1887,0.1684]$} & {$[0.2181,0.1908]$} \\
\hline Alternative $19\left(\mathrm{FM}_{19}\right)$ & {$[0.0320,0.0248]$} & {$[0.0292,0.0212]$} & {$[0.0510,0.0431]$} & {$[0.2181,0.1908]$} \\
\hline Alternative $20\left(\mathrm{FM}_{20}\right)$ & {$[0.0213,0.0172]$} & {$[0.0292,0.0212]$} & {$[0.1198,0.1058]$} & {$[0.2181,0.1908]$} \\
\hline Alternative $21\left(\mathrm{FM}_{21}\right)$ & {$[-0.0001,0.0019]$} & {$[0.0292,0.0212]$} & {$[-0.1556,-0.1449]$} & {$[-0.1588,-0.1954]$} \\
\hline Alternative $22\left(\mathrm{FM}_{22}\right)$ & {$[0.0320,0.0248]$} & {$[0.0043,0.0064]$} & {$[-0.0867,-0.0822]$} & {$[-0.0174,-0.0023]$} \\
\hline Alternative $23\left(\mathrm{FM}_{23}\right)$ & {$[0.0427,0.0324]$} & {$[-0.0122,-0.0083]$} & {$[-0.0179,-0.0196]$} & {$[-0.1117,-0.0988]$} \\
\hline Alternative $24\left(\mathrm{FM}_{24}\right)$ & {$[0.0427,0.0324]$} & {$[-0.0040,-0.0009]$} & {$[-0.1556,-0.1449]$} & {$[-0.1588,-0.1471]$} \\
\hline Alternative $25\left(\mathrm{FM}_{25}\right)$ & {$[0.0534,0.0400]$} & {$[0.0209,0.0212]$} & {$[0.0510,0.0431]$} & {$[-0.1117,-0.0988]$} \\
\hline
\end{tabular}

TABLE 16: Closeness coefficient (CC) and ranking of the failure modes.

\begin{tabular}{|c|c|c|}
\hline Alternatives & $\mathrm{CCi}$ & Rank \\
\hline Alternative $1\left(\mathrm{FM}_{1}\right)$ & 0.6500 & 6 \\
\hline Alternative $2\left(\mathrm{FM}_{2}\right)$ & 0.5722 & 8 \\
\hline Alternative $3\left(\mathrm{FM}_{3}\right)$ & 0.3944 & 10 \\
\hline Alternative $4\left(\mathrm{FM}_{4}\right)$ & 0.9681 & 1 \\
\hline Alternative $5\left(\mathrm{FM}_{5}\right)$ & -0.1310 & 16 \\
\hline Alternative $6\left(\mathrm{FM}_{6}\right)$ & -0.2845 & 18 \\
\hline Alternative $7\left(\mathrm{FM}_{7}\right)$ & -0.0424 & 13 \\
\hline Alternative $8\left(\mathrm{FM}_{8}\right)$ & -0.3572 & 19 \\
\hline Alternative $9\left(\mathrm{FM}_{9}\right)$ & 0.6531 & 5 \\
\hline Alternative $10\left(\mathrm{FM}_{10}\right)$ & 0.5715 & 9 \\
\hline Alternative $11\left(\mathrm{FM}_{11}\right)$ & -0.4926 & 20 \\
\hline Alternative $12\left(\mathrm{FM}_{12}\right)$ & -0.8961 & 25 \\
\hline Alternative $13\left(\mathrm{FM}_{13}\right)$ & -0.1079 & 14 \\
\hline Alternative $14\left(\mathrm{FM}_{14}\right)$ & 0.0393 & 11 \\
\hline Alternative $15\left(\mathrm{FM}_{15}\right)$ & -0.7230 & 24 \\
\hline Alternative $16\left(\mathrm{FM}_{16}\right)$ & 0.9314 & 2 \\
\hline Alternative $17\left(\mathrm{FM}_{17}\right)$ & -0.5610 & 22 \\
\hline Alternative $18\left(\mathrm{FM}_{18}\right)$ & 0.8182 & 3 \\
\hline Alternative $19\left(\mathrm{FM}_{19}\right)$ & 0.6101 & 7 \\
\hline Alternative $20\left(\mathrm{FM}_{20}\right)$ & 0.7233 & 4 \\
\hline Alternative $21\left(\mathrm{FM}_{21}\right)$ & -0.6024 & 23 \\
\hline Alternative $22\left(\mathrm{FM}_{22}\right)$ & -0.1212 & 15 \\
\hline Alternative $23\left(\mathrm{FM}_{23}\right)$ & -0.1934 & 17 \\
\hline Alternative $24\left(\mathrm{FM}_{24}\right)$ & -0.5362 & 21 \\
\hline Alternative $25\left(\mathrm{FM}_{25}\right)$ & 0.0190 & 12 \\
\hline
\end{tabular}




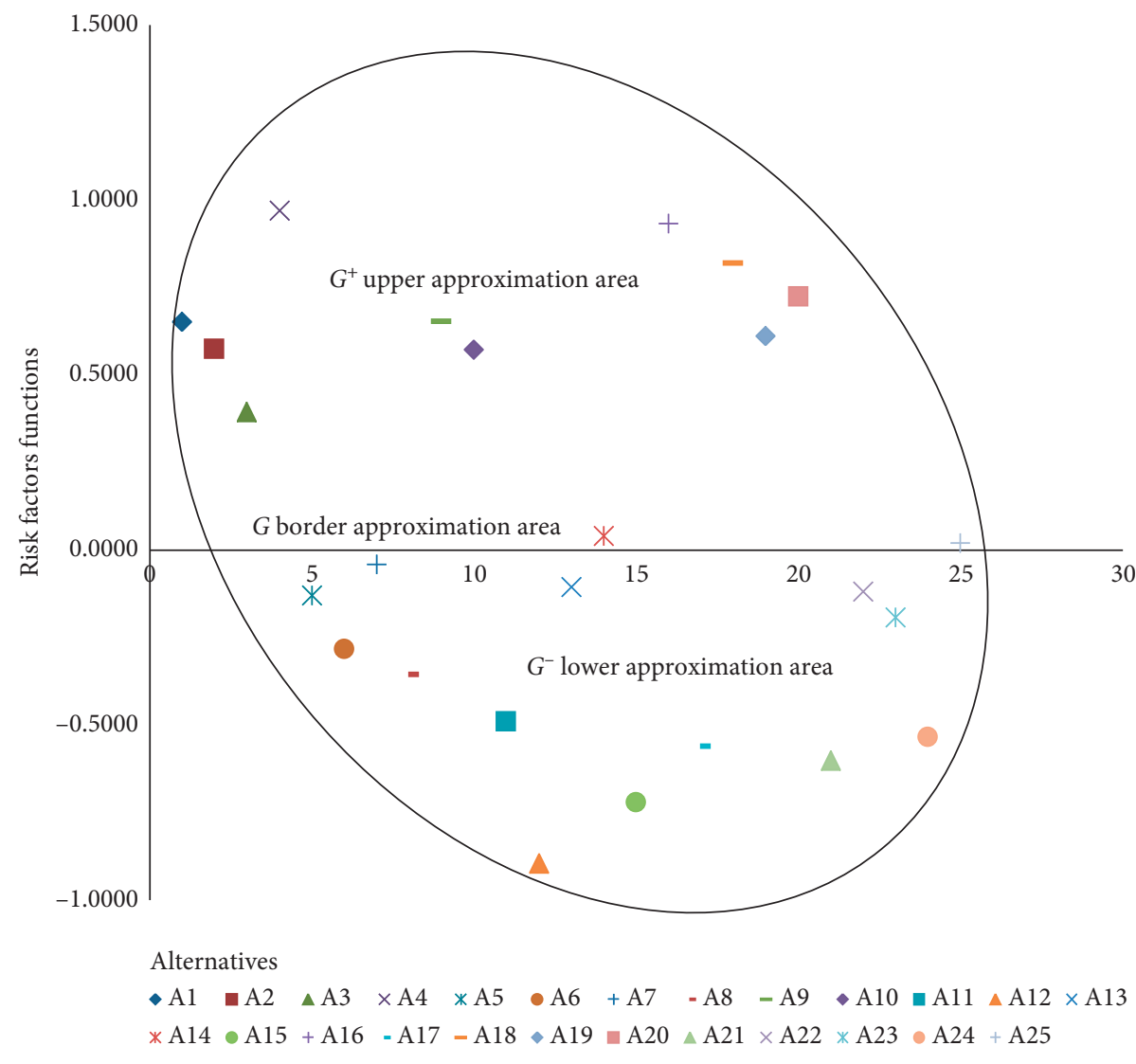

FIGURE 5: Grey MABAC ranking results.

TABLE 17: Summary of the comparative analysis.

$\begin{array}{cc}\text { Most critical } & \text { Less critical } \\ \mathrm{FM}_{25}>\mathrm{FM}_{19}>\mathrm{FM}_{22} & - \\ \mathrm{FM}_{12}<\mathrm{FM}_{8}<\mathrm{FM}_{21} & \mathrm{FM}_{9}>\mathrm{FM}_{16}>\mathrm{FM}_{4} \\ \mathrm{FM}_{12}<\mathrm{FM}_{15}<\mathrm{FM}_{21} & \mathrm{FM}_{4}>\mathrm{FM}_{16}>\mathrm{FM}_{18}\end{array}$

appropriate value of criteria $n$. To move to the next pace, the consistency rate of the study must be minor than 0.1 .

$$
\begin{aligned}
Q_{i j} & =\frac{1}{2} \times\left(\underline{Q_{i j}}+\overline{Q_{i j}}\right), \\
\mathrm{CI} & =\frac{\left(\nabla_{\max }-n\right)}{(n-1)}, \\
\mathrm{CR} & =\left(\frac{\mathrm{CI}}{\mathrm{RI}}\right) .
\end{aligned}
$$

Step 3: normalization of the grey weights: the grey row sums $\left(\otimes S_{i}\right)$ are normalized by applying equations (13), (15), and (16) given below to determine the final grey weights $\left(\otimes x_{i}\right)$ of criterion, respectively. The obtained grey values are subsequently integrated with the grey MABAC method to rank the alternatives.

$$
\begin{aligned}
\otimes S_{i} & =\sum_{j=1}^{n}\left[\underline{Q_{i j}}, \overline{Q_{i j}}\right], \\
\otimes S_{i} & =\left[\underline{S_{i}}, \overline{S_{i}}\right], \\
\underline{S}_{i}^{*} & =\left[\frac{2 \times \underline{S_{i}}}{\sum_{i=1}^{n} \underline{S_{i}}+\sum_{i=1}^{n} \overline{S_{i}}}\right], \\
\overline{S_{i}^{*}} & =\left[\frac{2 \times \overline{S_{i}}}{\sum_{i=1}^{n} \underline{S_{i}}+\sum_{i=1}^{n} \overline{S_{i}}}\right], \\
\otimes X_{i} & =\left[\underline{S_{i}^{*}}, \overline{S_{i}^{*}}\right]=\left[\underline{x_{i}}, \overline{x_{i}}\right] .
\end{aligned}
$$

4.3. Grey MABAC Model for the Failure Modes Prioritizing. After obtaining the weights coefficients from the grey AHP model, the ground is ready to develop the mathematical equations of the grey MABAC model. The process of 
TABle 18: Comparative analysis results.

\begin{tabular}{|c|c|c|c|c|c|c|}
\hline \multirow{3}{*}{ Alternatives } & \multicolumn{6}{|c|}{ Approaches } \\
\hline & \multicolumn{2}{|c|}{ Traditional RPN } & \multicolumn{2}{|c|}{$\begin{array}{l}\text { Traditional RPN with the } \\
\text { grey model }\end{array}$} & \multicolumn{2}{|c|}{$\begin{array}{c}\text { Proposed RPN with the } \\
\text { grey model }\end{array}$} \\
\hline & $(\mathrm{O} \times \mathrm{S} \times \mathrm{D})$ & Rank & $\mathrm{CCi}$ & Rank & $\mathrm{CCi}$ & Rank \\
\hline Alternative $1\left(\mathrm{FM}_{1}\right)$ & 72 & 14 & 0.1132 & 7 & 0.6500 & 6 \\
\hline Alternative $2\left(\mathrm{FM}_{2}\right)$ & 20 & 20 & 0.1910 & 5 & 0.5722 & 8 \\
\hline Alternative $3\left(\mathrm{FM}_{3}\right)$ & 150 & 10 & 0.0386 & 9 & 0.3944 & 10 \\
\hline Alternative $4\left(\mathrm{FM}_{4}\right)$ & 9 & 22 & 0.2446 & 3 & 0.9681 & 1 \\
\hline Alternative $5\left(\mathrm{FM}_{5}\right)$ & 5 & 25 & -0.0887 & 21 & -0.1310 & 16 \\
\hline Alternative $6\left(\mathrm{FM}_{6}\right)$ & 175 & 8 & -0.0336 & 17 & -0.2845 & 18 \\
\hline Alternative $7\left(\mathrm{FM}_{7}\right)$ & 60 & 16 & -0.0078 & 15 & -0.0424 & 13 \\
\hline Alternative $8\left(\mathrm{FM}_{8}\right)$ & 120 & 11 & -0.2098 & 24 & -0.3572 & 19 \\
\hline Alternative $9\left(\mathrm{FM}_{9}\right)$ & 8 & 13 & 0.3357 & 1 & 0.6531 & 5 \\
\hline Alternative $10\left(\mathrm{FM}_{10}\right)$ & 36 & 19 & 0.2157 & 4 & 0.5715 & 9 \\
\hline Alternative $11\left(\mathrm{FM}_{11}\right)$ & 288 & 5 & 0.0079 & 12 & -0.4926 & 20 \\
\hline Alternative $12\left(\mathrm{FM}_{12}\right)$ & 9 & 22 & -0.2794 & 25 & -0.8961 & 25 \\
\hline Alternative $13\left(\mathrm{FM}_{13}\right)$ & 7 & 24 & -0.0680 & 20 & -0.1079 & 14 \\
\hline Alternative $14\left(\mathrm{FM}_{14}\right)$ & 12 & 21 & -0.0349 & 18 & 0.0393 & 11 \\
\hline Alternative $15\left(\mathrm{FM}_{15}\right)$ & 48 & 18 & 0.0460 & 8 & -0.7230 & 24 \\
\hline Alternative $16\left(\mathrm{FM}_{16}\right)$ & 60 & 16 & 0.2942 & 2 & 0.9314 & 2 \\
\hline Alternative $17\left(\mathrm{FM}_{17}\right)$ & 64 & 15 & -0.0606 & 19 & -0.5610 & 22 \\
\hline Alternative $18\left(\mathrm{FM}_{18}\right)$ & 120 & 11 & -0.1159 & 22 & 0.8182 & 3 \\
\hline Alternative $19\left(\mathrm{FM}_{19}\right)$ & 350 & 2 & 0.0016 & 13 & 0.6101 & 7 \\
\hline Alternative $20\left(\mathrm{FM}_{20}\right)$ & 240 & 6 & 0.0255 & 11 & 0.7233 & 4 \\
\hline Alternative $21\left(\mathrm{FM}_{21}\right)$ & 160 & 9 & -0.1311 & 23 & -0.6024 & 23 \\
\hline Alternative $22\left(\mathrm{FM}_{22}\right)$ & 336 & 3 & 0.0006 & 14 & -0.1212 & 15 \\
\hline Alternative $23\left(\mathrm{FM}_{23}\right)$ & 192 & 7 & 0.1881 & 6 & -0.1934 & 17 \\
\hline Alternative $24\left(\mathrm{FM}_{24}\right)$ & 320 & 4 & 0.0276 & 10 & -0.5362 & 21 \\
\hline Alternative $25\left(\mathrm{FM}_{25}\right)$ & 360 & 1 & -0.0318 & 16 & 0.0190 & 12 \\
\hline
\end{tabular}

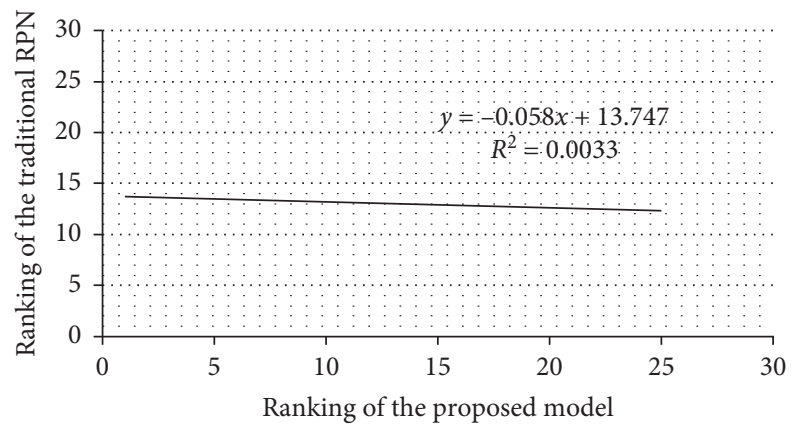

Figure 6: Negative correlation.

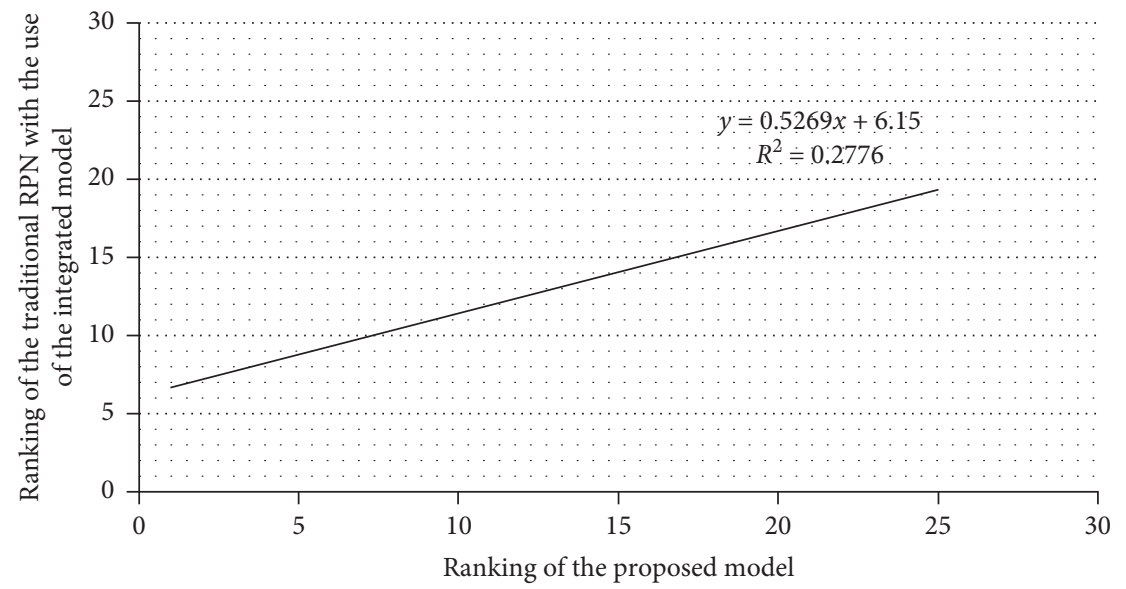

Figure 7: Positive correlation. 
TABLE 19: Diverse scenarios of risk factors weights.

\begin{tabular}{lccccc}
\hline RPN criteria & Original & Scenario 1 & Criteria weights scenarios & Scenario 2 & Scenario 3 \\
\hline C1 (occurrence) & 0.0679 & 0.0793 & 0.0788 & 1061 & 0.0774 \\
C1 (severity) & 0.0589 & 0.0516 & 0.0718 & 0.0783 & 0.0699 \\
C3 (detection) & 0.4916 & 0.4896 & 0.2416 & 0.2696 & 0.2154 \\
C4 (expected cost) & 0.3815 & 0.3795 & 0.6078 & 0.5461 & 0.6373 \\
\hline
\end{tabular}

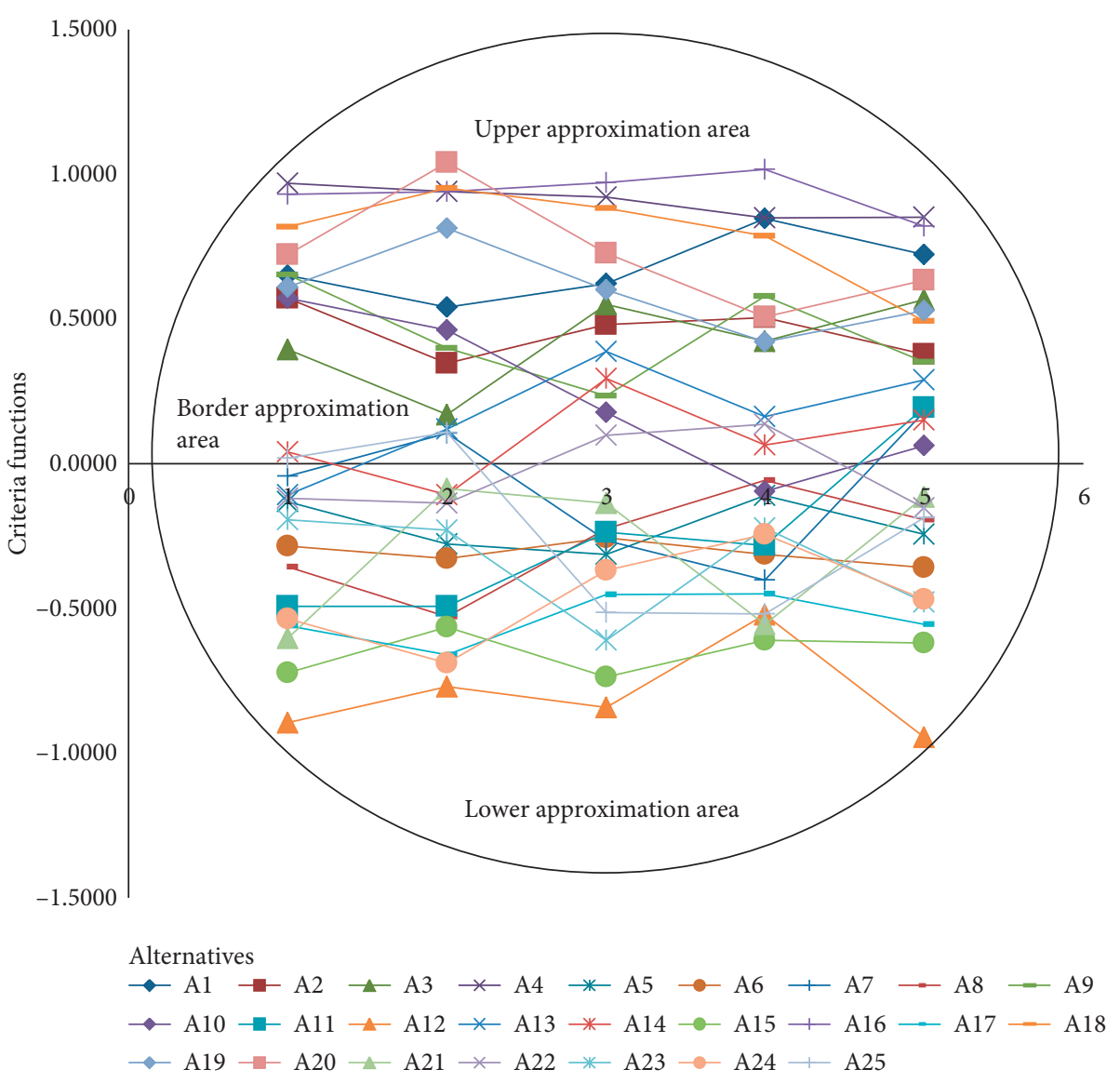

FIGURE 8: Developed model sensitivity analysis.

applying the grey MABAC model consists of seven main paces as given below:

Step 1: generating the initial decision matrix $(\widehat{T})$ : the research problem considers $m$ number of alternatives $\left(A_{i}, i=1,2,3, \ldots, m\right)$ and $n$ number of
$\operatorname{criteria}\left(C_{j}, j=1,2,3, \ldots, n\right)$. Here, $\widehat{T}^{k}=\left[\otimes t_{i j}^{k}\right]_{m \times n}$ denotes the grey decision matrix generated by the decision-maker $R_{k}$ and with the support of Tables 4-6.

$$
\begin{aligned}
& \widehat{T}^{k}=\left[\otimes t_{i j}^{k}\right]_{m \times n}
\end{aligned}
$$

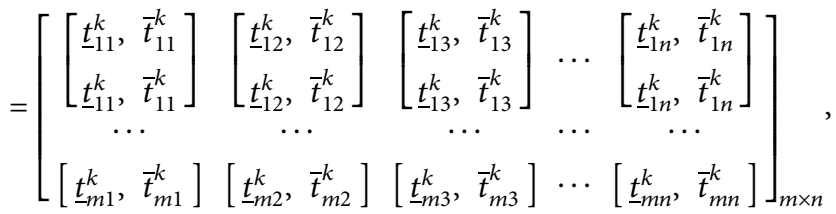


TABLE 20: Ranking results based on diverse scenarios of risk factors weights.

\begin{tabular}{|c|c|c|c|c|c|}
\hline \multirow[b]{2}{*}{ Failure modes } & \multicolumn{5}{|c|}{ Alternatives ranking response scenarios } \\
\hline & $\begin{array}{c}\text { Original } \\
\text { CCi (rank) }\end{array}$ & $\begin{array}{l}\text { Scenario } 1 \\
\text { CCi (rank) }\end{array}$ & $\begin{array}{l}\text { Scenario } 2 \\
\text { CCi (rank) }\end{array}$ & $\begin{array}{l}\text { Scenario } 3 \\
\text { CCi (rank) }\end{array}$ & $\begin{array}{l}\text { Scenario } 4 \\
\text { CCi (rank) }\end{array}$ \\
\hline $\mathrm{A} 1\left(\mathrm{FM}_{1}\right)$ & $0.6500(6)$ & $0.5410(6)$ & $0.6217(5)$ & $0.8459(3)$ & $0.7212(3)$ \\
\hline A2 $\left(\mathrm{FM}_{2}\right)$ & $0.5722(8)$ & $0.3467(9)$ & $0.4794(8)$ & $0.5050(7)$ & $0.3772(8)$ \\
\hline A3 $\left(\mathrm{FM}_{3}\right)$ & $0.3944(10)$ & $0.1699(10)$ & $0.5511(7)$ & $0.4236(8)$ & $0.5660(5)$ \\
\hline $\mathrm{A} 4\left(\mathrm{FM}_{4}\right)$ & $0.9681(1)$ & $0.9408(3)$ & $0.9203(2)$ & $0.8487(2)$ & $0.8526(1)$ \\
\hline A5 $\left(\mathrm{FM}_{5}\right)$ & $-0.1310(16)$ & $-0.2778(18)$ & $-0.3132(19)$ & $-0.1106(15)$ & $-0.2436(19)$ \\
\hline A6 $\left(\mathrm{FM}_{6}\right)$ & $-0.2845(18)$ & $-0.3288(19)$ & $-0.2572(17)$ & $-0.3148(19)$ & $-0.3591(20)$ \\
\hline A7 $\left(\mathrm{FM}_{7}\right)$ & $-0.0424(13)$ & $0.1055(13)$ & $-0.2655(18)$ & $.4036(20)$ & $0.1784(12)$ \\
\hline $\mathrm{A} 8\left(\mathrm{FM}_{8}\right)$ & $-0.3572(19)$ & $-0.5281(21)$ & $-0.2248(15)$ & $-0.0571(13)$ & $-0.1942(18)$ \\
\hline A9 $\left(\mathrm{FM}_{9}\right)$ & $0.6531(5)$ & $0.3987(8)$ & $0.2345(11)$ & $0.5798(5)$ & 0.3513 (9) \\
\hline A10 $\left(\mathrm{FM}_{10}\right)$ & $0.5715(9)$ & $0.4606(7)$ & $0.1760(12)$ & $-0.0957(14)$ & $0.0608(14)$ \\
\hline A11 $\left(\mathrm{FM}_{11}\right)$ & $-0.4926(20)$ & $-0.4923(20)$ & $-0.2375(16)$ & $.2835(18)$ & $0.1942(11)$ \\
\hline A12 $\left(\mathrm{FM}_{12}\right)$ & $-0.8961(25)$ & $-0.7710(25)$ & $-0.8425(25)$ & $-0.5213(23)$ & $-0.9461(25)$ \\
\hline A13 $\left(\mathrm{FM}_{13}\right)$ & $-0.1079(14)$ & $0.1182(11)$ & 0.3861 (9) & $1624(10)$ & $0.2889(10)$ \\
\hline A14 $\left(\mathrm{FM}_{14}\right)$ & $0.0393(11)$ & $-0.1073(15)$ & $0.2939(10)$ & $0646(12)$ & $0.1514(13)$ \\
\hline A15 $\left(\mathrm{FM}_{15}\right)$ & $-0.7230(24)$ & $-0.5652(22)$ & $-0.7375(24)$ & $-0.6103(25)$ & $-0.6206(24)$ \\
\hline A16 $\left(\mathrm{FM}_{16}\right)$ & $0.9314(2)$ & $0.9408(3)$ & $0.9718(1)$ & $1.0169(1)$ & $0.8216(2)$ \\
\hline $\mathrm{A} 17\left(\mathrm{FM}_{17}\right)$ & $-0.5610(22)$ & $-0.6616(23)$ & $-0.4534(21)$ & $-0.4502(21)$ & $-0.5567(23)$ \\
\hline A18 $\left(\mathrm{FM}_{18}\right)$ & $0.8182(3)$ & $0.95319(2)$ & $0.8830(3)$ & $0.7857(4)$ & $0.4921(7)$ \\
\hline A19 $\left(\mathrm{FM}_{19}\right)$ & $0.6101(7)$ & $0.8132(5)$ & $0.5993(6)$ & $0.4205(9)$ & $0.5290(6)$ \\
\hline A20 $\left(\mathrm{FM}_{20}\right)$ & $0.7233(4)$ & $1.0399(1)$ & $0.7262(4)$ & $0.5063(6)$ & $0.6335(4)$ \\
\hline A21 $\left(\mathrm{FM}_{21}\right)$ & $-0.6024(23)$ & $-0.08569(14)$ & $-0.1375(14)$ & $-0.5565(24)$ & $-0.1154(15)$ \\
\hline A22 $\left(\mathrm{FM}_{22}\right)$ & $-0.1212(15)$ & $-0.1358(16)$ & 0.0970 (13) & 0.1355 (11) & $-0.1543(16)$ \\
\hline A23 $\left(\mathrm{FM}_{23}\right)$ & $-0.1934(17)$ & $-0.2310(17)$ & $-0.6102(23)$ & $-0.2233(16)$ & -0.4785 \\
\hline A24 $\left(\mathrm{FM}_{24}\right)$ & $-0.5362(21)$ & $-0.6884(24)$ & $-0.3692(20)$ & $-0.2452(17)$ & $-0.4684(21)$ \\
\hline A25 $\left(\mathrm{FM}_{25}\right)$ & $0.0190(12)$ & $0.1061(12)$ & $-0.5139(22)$ & $-0.5204(22)$ & $-0.1867(17)$ \\
\hline
\end{tabular}

where $\otimes t_{i j}^{k}$ denotes the performance grade of $A_{i}$ with respect to criterion $C_{j}$ according to $R_{k}(k=1,2,3, \ldots, K)$. Thus, the cross-functional decision-makers $K$ are involved in the evaluation procedure. Each $R s$ is given equal importance $\varepsilon_{\mathbf{k}}$ (where $\sum_{k-1}^{K} \alpha_{k}=1$ ). Then, the grey systems theory is applied to handle the fuzziness of the collected data. The linguistic variables and the grey scale for the four risk factors are given in Tables 4-7.
Step 2: formation of the grey decision matrix $(\widehat{T})$ : the decision matrices are gathered from the cross-functional decision-makers to aggregate the initial decision matrices $\widehat{T}^{k}(k=1,2,3, \ldots, K)$ into a grey decision matrix set $\widehat{T}=\left[\otimes t_{i j}\right]_{m \times n}$ as follows:

$$
\begin{aligned}
\widehat{T}^{k} & =\left[\otimes t_{i j}\right]_{m \times n} \\
& =\left[\begin{array}{ccccc}
{\left[\underline{t}_{11}, \bar{t}_{11}\right]} & {\left[\underline{t}_{12}, \bar{t}_{12}\right]} & {\left[\underline{t}_{13}, \bar{t}_{13}\right]} & \ldots & {\left[\underline{t}_{1 n}, \bar{t}_{1 n}\right]} \\
{\left[\underline{t}_{21}, \bar{t}_{21}\right]} & {\left[\underline{t}_{22}, \bar{t}_{22}\right]} & {\left[\underline{t}_{23}, \bar{t}_{23}\right]} & \ldots & {\left[\underline{t}_{2 n}, \bar{t}_{2 n}\right]} \\
{\left[\underline{t}_{31}, \bar{t}_{31}\right]} & {\left[\underline{t}_{32}, \bar{t}_{32}\right]} & {\left[\underline{t}_{33}, \bar{t}_{33}\right]} & \ldots & {\left[\underline{t}_{3 n}, \bar{t}_{3 n}\right]} \\
\ldots & \ldots & \ldots & \ldots & \ldots \\
{\left[\underline{t}_{m 1}, \bar{t}_{m 1}\right]} & {\left[\underline{t}_{m 2}, \bar{t}_{m 2}\right]} & {\left[\underline{t}_{m 3}, \bar{t}_{m 3}\right]} & \ldots & {\left[\underline{t}_{m n}, \bar{t}_{m n}\right]}
\end{array}\right]_{m \times n}, \\
\underline{t}_{i j} & =\sum_{k=1}^{K} \alpha_{k} \times \underline{t}_{i j}^{k}, \bar{t}_{i j}=\sum_{k=1}^{K} \alpha_{k} \times \bar{t}_{i j}^{k},
\end{aligned}
$$

where $m$ denotes the total number of the alternatives and $n$ denotes the total number of the criteria.
Step 3: normalization of the elements from the grey aggregated decision matrix $(\widehat{P})$ : the normalization of 
the elements of the grey aggregated matrix $(\widehat{P})$ are identified from the initial matrix $\widehat{T}$ applying the following equation:

$$
\otimes p_{i j}=\left[\underline{p}_{i j}, \bar{p}_{i j}\right]= \begin{cases}\frac{\underline{t}_{i j}}{t_{j}^{+}}, & \frac{\bar{t}_{i j}}{t_{j}^{+}}, \\ \frac{t_{j}^{-}}{\bar{t}_{i j}}, & \frac{t_{j}^{-}}{\underline{t}_{i j}},\end{cases}
$$

where $t_{j}^{+}=\max _{1 \leq i \leq m}\left(\bar{t}_{i j}\right)$ denotes the benefit category criteria where maximal value of the criterion is required, while $t_{j}^{-}=\min _{1 \leq i \leq m}\left(\underline{t}_{i j}\right)$ indicates the cost category criteria where the minimum value of the criterion is required. Therefore, the normalized grey decision matrix is illustrated as follows:

$$
\begin{aligned}
& \widehat{P}=\left[\otimes t_{i j}\right]_{m \times n} \\
& =\left[\begin{array}{ccccc}
{\left[\underline{p}_{11}, \bar{p}_{11}\right]} & {\left[\underline{p}_{12}, \bar{p}_{12}\right]} & {\left[\underline{p}_{13}, \bar{p}_{13}\right]} & \cdots & {\left[\underline{t}_{1 n}, \bar{t}_{1 n}\right]} \\
{\left[\underline{p}_{21}, \bar{p}_{21}\right]} & {\left[\underline{p}_{22}, \bar{p}_{22}\right]} & {\left[\underline{p}_{23}, \bar{p}_{23}\right]} & \cdots & {\left[\underline{p}_{2 n}, \bar{p}_{2 n}\right]} \\
{\left[\underline{p}_{31}, \bar{p}_{31}\right]} & {\left[\underline{p}_{32}, \bar{p}_{32}\right]} & {\left[\underline{p}_{33}, \bar{p}_{33}\right]} & \cdots & {\left[\underline{p}_{3 n}, \bar{p}_{3 n}\right]} \\
\ldots & \ldots & \ldots & \cdots & \cdots \\
{\left[\underline{p}_{m 1}, \bar{p}_{m 1}\right]} & {\left[\underline{p}_{m 2}, \bar{p}_{m 2}\right]} & {\left[\underline{p}_{m 3}, \bar{p}_{m 3}\right]} & \cdots & {\left[\underline{p}_{m n}, \bar{p}_{m n}\right]}
\end{array}\right]_{m \times n}
\end{aligned}
$$

Step 4: computation of the elements from the greyweighted decision mrix $(\widehat{G})$ : the elements of the weighted matrix $(\widehat{G})$ are computed based on equation (23). Thus, $W_{j}$ denotes the weighted coefficients of the criterion $j$. By applying equation (24), the weighted matrix $(\widehat{G})$ is formulated as follows:

$$
\begin{aligned}
& \otimes g_{i j}=\left[\underline{g}_{i j}, \bar{g}_{i j}\right]=W_{j} \times \otimes p_{i j}=\left[W_{j} \times \underline{p}_{11}, W_{j} \times \bar{p}_{11}\right] \text {, } \\
& \widehat{G}=\left[\otimes g_{i j}\right]_{m \times n}
\end{aligned}
$$

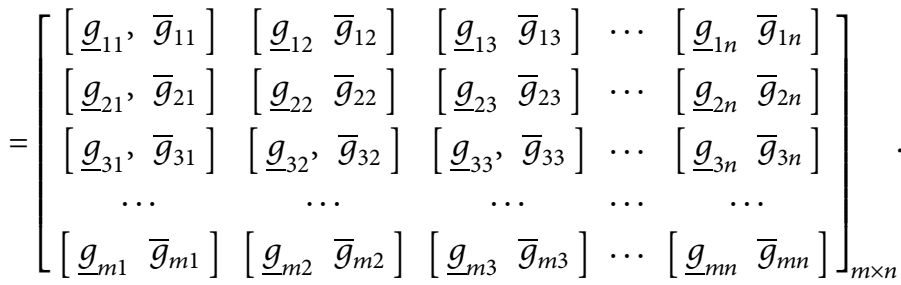

Step 5: calculation of the grey Border Approximation Area (BAA) matrix $(\widehat{B})$ : the grey Border Approximation Area (BAA) for each criterion is calculated by using equation (25):

$\otimes b_{i j}=\left[\underline{b}_{j}, \bar{b}_{j}\right]=\left[\left(\prod_{i=1}^{m} \underline{g}_{i j}\right)^{1 / m},\left(\prod_{i=1}^{m} \bar{g}_{i j}\right)^{1 / m}\right]$.
Thus, $\left[\underline{g}_{i j}, \bar{g}_{i j}\right]$ denotes the elements of the weighted matrix $(\widehat{G})$ and $m$ indicates the total number of alternatives. Once the grey value $\otimes b_{i j}=\left[\underline{b}_{j}, \bar{b}_{j}\right]$ is calculated for each criterion, a border approximation area vector is generated. However, the BAA is an orientation point for each alternative. Furthermore, the grey vector $\vec{b}=\left(\otimes g_{1}, \otimes g_{2}, \otimes g_{3}, \ldots, \otimes g_{n}\right)_{1 \times n}$ is used in the grey Border Approximation Area (BAA) matrix $(\widehat{B})$, as rows of the following matrix: 


$$
\widehat{B}=\left[\begin{array}{ccccc}
{\left[\underline{b}_{1}, \bar{b}_{1}\right]} & {\left[\underline{b}_{2}, \bar{b}_{2}\right]} & {\left[\underline{b}_{3}, \bar{b}_{3}\right]} & \cdots & {\left[\underline{b}_{n}, \bar{b}_{n}\right]} \\
{\left[\underline{b}_{1}, \bar{b}_{1}\right]} & {\left[\underline{b}_{2}, \bar{b}_{2}\right]} & {\left[\underline{b}_{3}, \bar{b}_{3}\right]} & \cdots & {\left[\underline{b}_{n}, \bar{b}_{n}\right]} \\
{\left[\underline{b}_{1}, \bar{b}_{1}\right]} & {\left[\underline{b}_{2}, \bar{b}_{2}\right]} & {\left[\underline{b}_{3}, \bar{b}_{3}\right]} & \cdots & {\left[\underline{b}_{n}, \bar{b}_{n}\right]} \\
\cdots & \cdots & \cdots & \cdots & \cdots \\
{\left[\underline{b}_{1}, \bar{b}_{1}\right]} & {\left[\underline{b}_{2}, \bar{b}_{2}\right]} & {\left[\underline{b}_{2}, \bar{b}_{2}\right]} & \cdots & {\left[\underline{b}_{n}, \bar{b}_{n}\right]}
\end{array}\right]_{m \times n}
$$

Step 6: computation of the alternatives distance from the BAA matrix for the matrix elements $(X)$ :

The distance of the alternatives is calculated employing the "Euclidean distance" between the grey numbers $\otimes g_{i j}$ and $\otimes b_{j}$. Then, the elements matrix $X$ is carried out as follows:

$$
X=\widehat{G}-\widehat{B}=\left[x_{i j}\right]_{m \times n}=\left[\begin{array}{ccccc}
\otimes g_{11}-\otimes b_{1} & \otimes g_{12}-\otimes b_{2} & \otimes g_{13}-\otimes b_{3} & \cdots & \otimes g_{1 n}-\otimes b_{n} \\
\otimes g_{21}-\otimes b_{1} & \otimes g_{22}-\otimes b_{2} & \otimes g_{23}-\otimes b_{3} & \cdots & \otimes g_{2 n}-\otimes b_{n} \\
\otimes g_{31}-\otimes b_{1} & \otimes g_{32}-\otimes b_{2} & \otimes g_{33}-\otimes b_{3} & \cdots & \otimes g_{3 n}-\otimes b_{n} \\
\cdots & \cdots & \cdots & \cdots & \cdots \\
\otimes g_{m 1}-\otimes b_{1} & \otimes g_{m 2}, \otimes b_{2} & \otimes g_{m 3}, \otimes b_{3} & \cdots & \otimes g_{m n}-\otimes b_{n}
\end{array}\right]_{m \times n}
$$

where

$$
X=\left[\begin{array}{ccccc}
x_{11} & x_{12} & x_{13} & \cdots & x_{1 n} \\
x_{21} & x_{22} & x_{23} & \cdots & x_{2 n} \\
x_{31} & x_{32} & x_{33} & \cdots & x_{3 n} \\
\cdots & \cdots & \cdots & \cdots & \cdots \\
x_{m 1} & x_{m 2} & x_{m 3} & \cdots & x_{m n}
\end{array}\right]
$$

The distance of the alternatives from the Border Approximation Area (BAA) of each criterion is defined as the difference between the elements in the grey-weighted matrix $(\widehat{G})$ and the value of the border approximation area $(\widehat{B})$. Therefore, $\otimes b_{j}$ denotes the border approximation area of criterion $C_{j}$, and $\otimes g_{i j}$ denotes the elements of the greyweighted matrix $(\widehat{G})$. The coefficient correlation is carried out as follows:

$$
\mathrm{CC}\left(A_{i}\right)=\sum_{j=1}^{n} x_{i j}, \quad i=1,2,3, \ldots, m, j=1,2,3, \ldots, n .
$$

Equation (30) denotes the sum of the distance of the alternatives from the Border Approximation Area $\left(b_{i j}\right)$. CC indicates the closeness coefficient of each alternative from the BAA. Alternative $A_{i}=(i=1,2,3, \ldots, m)$ belongs to the Border Approximation Area (BAA). There are three main areas and are pined as follows:

$$
A_{i} \in \begin{cases}G^{+}, & \text {if } x_{i j}>0, \text { upper approximation area, } \\ G, & \text { if } x_{i j}=0, \text { border approximation area, } \\ G^{-}, & \text {if } x_{i j}>0, \text { lower approximation area, }\end{cases}
$$

where $G^{+}$denotes the upper approximation area for alternatives that are equal or close to the ideal solution, meanwhile $G^{-}$denotes the lower approximation area for alternatives that are equal or close to the anti-ideal solution.

Step 7: ranking the Alternatives: the ranking of the alternatives is performed with the help of the following equation:

$$
\widehat{R}_{i}=\sum_{j=1}^{n} x_{i j}, \quad i=1,2,3, \ldots, j=1,2,3, \ldots
$$

\section{Case Study}

The proposed grey model is applied and verified within a multinational company of renewable energy production implemented in the north region of Morocco (Tangier and Tangier Med Port). The type of wind turbine that has been studied and selected for the risk assessment is "B63 with a 294 megawatts power rating and 63 meters long and 17 tonnes of weight." However, it is one of North Africa's largest and most powerful implanted power generation systems. In the present work, data have been collected from five cross-functional decision-makers $\left(R_{1}, R_{2}, R_{3}, R_{4}, R_{5}\right)$ who are working for the called organization with work experience ranging from 1 to 25 years and most of them have got postgraduation qualifications. As mentioned earlier, this paper focuses on four main risk factors explained in Figure 4.

5.1. Grey AHP Model Application. As stated earlier, the grey AHP model application for evaluating the risk priority entails three principal steps. The first step considers the 
construction of the grey pairwise comparison matrix. The second step is about normalizing the pairwise comparison matrix. The third step consists of the consistency index (CI) calculation. Then, compare the latter to the random index (RI) for the appropriate value of $n$.

Step 1: application: the grey comparison matrix is constructed with the support and the assessments of the qualified team. The grey comparison matrix is explained in detail in Table 8.

Step 2: application: the row sums of the grey comparison matrix and the grey weights of criteria of the proposed model are given in Table 9. The consistency rate $(\mathrm{CR})$ is calculated, and the random index for the four criteria is 0.90 .

Step 3: application: due to the space limits, only one application is provided. Table 9 presents the results of the Grey AHP model. However, the degree of consistency is satisfactory $\mathrm{CI} / \mathrm{RI}=0.05638$. The rate indicates that the collected data from decision-makers is reliable and feasible.

5.2. GMABAC Model Application. As mentioned above, the alternatives used in the present work range from the failure mode $\left(\mathrm{FM}_{1}\right)$ to $\left(\mathrm{FM}_{25}\right)$. The assessment of the alternatives in the grey MABAC is related to the four risk factors. The obtained weights of the four risk factors are integrated with grey MABAC to rank the critical failure modes in the wind turbine. However, Table 10 presents the performance ratings of the five cross-functional decision-makers .

Step 1 and 2: applications: the linguistic terms are translated into a grey number and presented in Table 11 . The aggregated grey decision matrix is obtained by applying equations (18)-(20).

Step 3: application: the aggregated grey decision matrix $(\widehat{T})$ is converted into the normalized grey decision matrix $(\widehat{P})$ by using equations $(21)$ and $(22)$. Table 12 illustrates the normalized grey decision matrix $(\widehat{P})$.

Step 4: application: the weighted grey decision matrix $(\widehat{G})$ is computed by using the weight vector and equations (19) and (20) However, the weighted matrix is given as presented in Table 13.

Step 5: application: this step considers the calculation of the Boer Appoximation Area (BAA) by applying equations (25) and (26). Therefore, the grey BAA matrix $(\widehat{B})$ is performed in this work in order to precisely determine the criticality of the failure modes in the wind turbine. The latter is calculated with the help of the geometric average as per shown in Table 14.

Step 6: application: the preference index matrix $(X)$ is calculated by utilizing equation (27) as per viewed in Table 15. However, the obtained results are divided into two categories. The negative values denote the weakness (critical failures modes), and the positive values refer to the strength (noncritical failure modes) in the wind turbine assembly.
Step 7: application: the closeness coefficients for each failure mode (CC) are calculated by applying equation (29) and given in Table 16. Furthermore, the failure modes are ranked to the descending order (from the upper value to the lowest value). However, the obtained results through the application of the proposed grey model reveal the inherent risk in the wind turbine where $\mathrm{FM}_{4}$ (fracture in the shell) is less critical than $\left(\mathrm{FM}_{16}\right.$ gearbox malfunction) and which in its turn is less critical than $\left(\mathrm{FM}_{18}\right.$ excessive fatigue of the generator); meanwhile, the failure mode ( $\mathrm{FM}_{12}$ main shaft vibration) is the most critical part in the wind turbine assembly.

A graphical representation of the closeness coefficients is necessary to complete the grey model application. Figure 5 illustrates the final results of the grey AHP-MABAC model. However the failure modes which belong to the upper approximation area $\mathrm{G}^{+}$are noncritical failures (high-performance components/parts), while the failure modes which belong to the lower approximation area $G^{-}$are the most critical in the system (weak components/parts).

\section{Results and Discussions}

A comparative analysis of the three different approaches is furnished in the first place. Then, the consequences of the sensitivity analysis of the proposed grey model are presented.

6.1. Comparative Analysis. A comparison of failure modes ranking utilizing different approaches is conducted in this present paper to demonstrate the practicability of the proposed grey integrated model. First, the traditional RPN approach is applied by multiplying the three classical risk factors, namely, 'occurrence, severity, and detection,' with the support of the cross-functional decision-makers. Second, the traditional RPN is applied by using the proposed grey integrated model. Third, the proposed RPN approach is applied to the use of the proposed grey integrated model. The ranking results of the three different approaches are presented in Tables 17 and 18. Here, the results validate the statistical significance of the grey model among the ranking acquired through the application of different methods. Subsequently, Pearson's correlation coefficient [63] is used to compare mutual correspondence between the three approaches. Here, the obtained results validate the statistical significance of the grey risk-based model among the ranking acquired through the application of different methods. The comparative analysis explanation of the three approaches is as follows:

(i) Traditional RPN approach: the most critical failure modes in the wind turbine, according to the first comparative approach, are ranked as follows $\mathrm{FM}_{12}>\mathrm{FM}_{8}>\mathrm{FM}_{21}$. The details are presented in Table 18. Figure 6 shows the negative correlation between the first comparative approach and the third comparative approach. The negative correlation affirms that the ranking order of both approaches moves in the opposite direction. 
(ii) Grey integrated model with the traditional RPN approach: the most critical failure modes in the wind turbine, according to the second comparative approach, are ranked as follows: $\mathrm{FM}_{12}<\mathrm{FM}_{8}<\mathrm{FM}_{21}$. The first worst-ranked failure mode in this comparative approach is the main shaft vibration. Next, the second worst-ranked failure mode is Pitch system excessive loading. Then, the third worse-ranked failure mode is a frequency converter set. Meanwhile, the less-critical failure modes are $\mathrm{FM}_{9}>\mathrm{FM}_{16}>\mathrm{FM}_{4}$. Figure 7 shows the positive correlation between the second comparative approach and the third comparative approach. The positive correlation affirms that the ranking order of both approaches moves in the same direction. The details are presented in Table 18.

(iii) Grey integrated model with the proposed RPN Approach: the most critical failure modes in the wind turbine, according to the third comparative approach, are ranked as follows: $\mathrm{FM}_{12}<\mathrm{FM}_{15}<\mathrm{FM}_{21}$. The first worst-ranked failure mode in the study is the main shaft set which has a negative critical effect on the remaining parts of the system. Thus, the second worstranked failure mode is the gearbox vibration which leads to the collapse of the assembly for a long time. Hence, the third worse-ranked failure mode is the frequency converter set that restrains the conversion of the energy to the transformer which in turn plugs the power generation and causes economic damages to the company. Meanwhile, the less-critical failure modes are $\mathrm{FM}_{4}>\mathrm{FM}_{16}>\mathrm{FM}_{18}$. The $\mathrm{FM}_{4}$ (the fracture in the shell) is less critical than the $\mathrm{FM}_{16}$ (the gearbox malfunction) which in turn is less critical than the $\mathrm{FM}_{18}$ (the excessive fatigue of the generator). The details are presented in Table 18.

6.2. Sensitivity Analysis. The alternatives ranking results are related to the criteria weight coefficients. Thus, it is necessary to conduct a sensitivity analysis by changing the weight coefficients of the criteria to test the reliability and practicability of the proposed methodology. However, the alteration in criteria weight coefficients drives soft changes in the ranking order of the alternatives. Based on that, a sensitivity analysis is conducted in the present paper to control the unity amongst the risk factor criteria "occurrence, severity, detection, and expected costs," observing the diversification in failure modes ranking with the diversification in criteria weights. The results of the sensitivity analysis for assessing the $n$ criteria weights $\left(C_{j}, j=1,2, \ldots, 4\right)$ are presented in Table 19, and their impact on the alternatives ranking order $\left(A_{i}, i=1,2, \ldots, 25\right)$ is given in Table 19 .

The consequences of the sensitivity analysis are shown in Figure 8. First, the results in Table 19 illustrate that the alteration in criteria weights $\left(C_{j}, j=1,2, \ldots, 4\right)$ throughout four different scenarios $\left\{S_{1}-S_{4}\right\}$ affect the ranking order of the alternatives. This changing demonstrates that the proposed approach is sensitive to modification in criteria weights. Second, the results of the failure modes ranking, as illustrated in Table 20, prove that $\mathrm{FM}_{12}$ is the first most critical failure mode and retains its rank in three scenarios $\left\{S_{1}, S_{2}, S_{4}\right\}$, while in scenario $\left\{S_{3}\right\}$, it is ranked the third most critical failure mode in the wind turbine. Third, the $\mathrm{FM}_{16}$ is the less-critical failure mode in the wind turbine, and it retains its rank in two scenarios $\left\{S_{2}, S_{3}\right\}$; meanwhile, in scenario $\left\{S_{4}\right\}$, it is ranked as the second most critical, and in scenario $\left\{S_{1}\right\}$, it is ranked as the third most critical.

However, it is observed that the ranking of the failure mode $\mathrm{FM}_{12}$ belongs to the lower approximation area which refers to the anti-ideal situation, while the failure mode $\mathrm{FM}_{16}$ belongs to the upper approximation area which refers to the ideal situation. The ranking of the failure modes in most of the scenarios remains steady unless some enormous modifications are made in criteria weights alteration. The sensitivity analysis conducted in the present work was meaningful to prioritize the failure modes for the wind turbine system.

\section{Conclusions}

The complex systems are designed to deliver the manufactured products, the delineated services and to have long life cycles to meet the user expectation. Renewable energy production is an interesting field entailing a large number of high technology complex systems. The inherent risks affect the economic activity of the organization, which in turn indirectly affects the productivity of the latter resulting in delays and insufficient energy. With these organizational perceptions, the effective solution to this challenge was to propose a grey multicriteria approach to support the FMECA. A novel RPN approach has been developed to overcome the results of the traditional RPN approach. However, the wind turbine has been selected as a convenient background to verify the proposed grey methodology. The grey AHP model has been applied to evaluate the relationship between the risk factors of the proposed RPN approach and calculate their weights. Then, the grey MABAC model has been applied to prioritize the most critical failure mode in the wind turbine. The weights generated from the grey AHP model has been employed as inputs in the grey MABAC model to assess the twenty-five failure modes.

In the traditional RPN approach, the failure modes evaluation is performed using crisp ratings, and the cross-functional decision-makers react inflexibly and imprecisely by using only white numbers. Thus, Pearson's correlation coefficient value of the classical RPN rating is negative which confirms that this method is not reliable. On the other hand, Pearson's correlation coefficient value of the proposed RPN approach with the support of the grey AHP-MABAC is positive which indicates that the proposed model is an imperative mechanism to envisage the interrelations among the severity, detection, occurrence, and expected costs criteria. This research study provides a series of significant contributions to the field of asset management:

(i) Grey systems theory (GST) is considered as a supportive tool to overcome the fuzziness and the uncertainty of poor data and small samples. 
Subsequently, it was capable in this work to drive and address ambiguous data weighting risk factors and alternatives in a systematic approach.

(ii) The grey AHP model has a foremost benefit due to its flexibility and certainty. It involves the consistency rate (CR) calculation, which was a compulsory phase during the model application process, to evaluate the reliability of the decision-maker's evaluation.

(iii) The grey MABAC model has a remarkable advantage over other MCDM techniques. It shows how to aggregate the decision-makers' judgements in a systematic approach. It allowed us to divide the criteria into beneficial (e.g., detecting the failure modes) and nonbeneficial (severity, occurrence, and expected costs of the failure modes). This model considers the calculation of the geometric average which was used to determine the performance results of a portfolio and/or an investment of the organization. Also, it considers the calculation of the closeness coefficient values (CCi) and categorized them into three different intervals (upper approximation area for positive values, border approximation area equal to zero, and lower approximation area negative values).

(iv) Graphical representation of the grey MABAC model will help the executive officials of the clean energy production management to appraise and select the most and the less-critical failure mode in the wind turbine. Unlike the traditional RPN, the proposed grey model highlights the mutual influences between the operational risk factors (severity-occurrence-detection) and the economic criteria (expected costs).

(v) Study findings are grounded on a single case institute (wind turbine type B63) and the judgements are assessed in rigorously. This helps managers from other national and international institutes to use this framework as a reference to analyze the inherent risks in the assemblies.

(iv) Integration of the engineering and management disciplines will allow managers to implement the most appropriate preventive solutions, implement the efficient reliability strategies for maintaining the system components in good work conditions, and enhance their designs in the future. With these organizational insights, the optimal investment strategy will have a positive impact needed for both the environment and the organization.

In future research, the proposed framework needs to be modified and includes the subcriteria considering hidden risks in the wind turbine and further be applied to other renewable energy production systems such as farm wind turbines and offshore wind turbines. Furthermore, numerous methods and MCDM techniques (based ANP, DEMATE, COPRAS, ARAS, grey connective maps, and D numbers) could be pragmatic to analyze such risk evaluation issues and support the relationship among the main and subcriteria.

\section{Data Availability}

The data used to support the findings of this study are included within the article.

\section{Conflicts of Interest}

The authors declare that they have no conflicts of interest.

\section{References}

[1] Y. Fu, X. Liu, and Z. Yuan, "Life-cycle assessment of multicrystalline photovoltaic (PV) systems in China," Journal of Cleaner Production, vol. 86, pp. 180-190, 2015.

[2] R. B. Santos and U. R. de Oliveira, "Analysis of occupational risk management tools for the film and television industry," International Journal of Industrial Ergonomics, vol. 72, pp. 199-211, 2019.

[3] T. Bjerga, T. Aven, and E. Zio, "Uncertainty treatment in risk analysis of complex systems: the cases of STAMP and FRAM," Reliability Engineering \& System Safety, vol. 156, pp. 203-209, 2016.

[4] F. Dinmohammadi and M. Shafiee, "A fuzzy-FMEA risk assessment approach for offshore wind turbines," International Journal of Prognostics and Health Management, vol. 4, no. 13, pp. 59-68, 2013.

[5] A. Zhou, D. Yu, and W. Zhang, "A research on intelligent fault diagnosis of wind turbines based on ontology and FMECA," Advanced Engineering Informatics, vol. 29, no. 1, pp. 115-125, 2015.

[6] M. G. Bharatbhai, "Failure mode and effect analysis of repower 5M wind turbine," International Journal of Advanced Scientific and Technical Research, vol. 2, no. 5, pp. 2394-2444, 2015.

[7] W. Song, X. Ming, Z. Wu, and B. Zhu, "Failure modes and effects analysis using integrated weight-based fuzzy TOPSIS," International Journal of Computer Integrated Manufacturing, vol. 26, no. 12, pp. 1172-1186, 2013.

[8] S. Carpitella, A. Certa, J. Izquierdo, and C. M. La Fata, "A combined multi-criteria approach to support FMECA analyses: a real-world case," Reliability Engineering \& System Safety, vol. 169, pp. 394-402, 2018.

[9] H.-W. Lo, J. J. H. Liou, C.-N. Huang, and Y.-C. Chuang, “A novel failure mode and effect analysis model for machine tool risk analysis," Reliability Engineering \& System Safety, vol. 183, pp. 173-183, 2019.

[10] L. Jun and X. Huibin, "Reliability analysis of aircraft equipment based on FMECA method," Physics Procedia, vol. 25, pp. 1816-1822, 2012.

[11] H.-C. Liu, L. Liu, and N. Liu, "Risk evaluation approaches in failure mode and effects analysis: a literature review," Expert Systems with Applications, vol. 40, no. 2, pp. 828-838, 2013.

[12] W. Faulkner and F. Badurdeen, "Sustainable value stream mapping (Sus-VSM): methodology to visualize and assess manufacturing sustainability performance," Journal of Cleaner Production, vol. 85, pp. 8-18, 2014.

[13] F. P. G. Márquez, A. M. Tobias, J. M. P. Pérez, and M. Papaelias, "Condition monitoring of wind turbines: techniques and methods," Renewable Energy, vol. 46, pp. 169-178, 2012. 
[14] A. Pillay and J. Wang, "Modified failure mode and effects analysis using approximate reasoning," Reliability Engineering \& System Safety, vol. 79, no. 1, pp. 69-85, 2003.

[15] S. M. Seyed-Hosseini, N. Safaei, and M. J. Asgharpour, "Reprioritization of failures in a system failure mode and effects analysis by decision making trial and evaluation laboratory technique," Reliability Engineering \& System Safety, vol. 91, no. 8, pp. 872-881, 2006.

[16] H. Gargama and S. K. Chaturvedi, "Criticality assessment models for failure mode effects and criticality analysis using fuzzy logic," IEEE Transactions on Reliability, vol. 60, no. 1, pp. 102-110, 2011.

[17] E. V. Kumar and S. K. Chaturvedi, "Prioritization of maintenance tasks on industrial equipment for reliability," International Journal of Quality \& Reliability Management, vol. 28, no. 1, pp. 109-126, 2011.

[18] G.-D. Li, D. Yamaguchi, and M. Nagai, "Application of greybased rough decision-making approach to suppliers selection," Journal of Modelling in Management, vol. 2, no. 2, pp. 131-142, 2007.

[19] H.-W. Lo and J. J. H. Liou, "A novel multiple-criteria decision-making-based FMEA model for risk assessment," Applied Soft Computing, vol. 73, pp. 684-696, 2018.

[20] M. Abolghasemi, V. Khodakarami, and H. Tehranifard, "A new approach for supply chain risk management: mapping SCOR into Bayesian network," Journal of Industrial Engineering and Management (JIEM), vol. 8, no. 1, pp. 280-302, 2015.

[21] N. J. Bahr, System Safety Engineering and Risk Assessment: A Practical Approach, CRC Press, Boca Raton, FL, USA, 2018.

[22] Y.-S. Lee, D.-J. Kim, J.-O. Kim, and H. Kim, "New FMECA methodology using structural importance and fuzzy theory," IEEE Transactions on Power Systems, vol. 26, no. 4, pp. 2364-2370, 2011.

[23] V. Holley, M. Jankovic, and B. Yannou, "Multiple-domain design scorecards: a method for architecture generation and evaluation through interface characterisation," vol. 23 , no. 1011, pp. 746-766, 2014

[24] H. Hwang, K. Lansey, and D. R. Quintanar, "Resilience-based failure mode effects and criticality analysis for regional water supply system," Journal of Hydroinformatics, vol. 17, no. 2, pp. 193-210, 2014.

[25] M. Braglia, "MAFMA: multi-attribute failure mode analysis," International Journal of Quality \& Reliability Management, vol. 17, no. 9, pp. 1017-1033, 2000.

[26] H.-C. Liu, L.-E. Wang, Z. Li, and Y.-P. Hu, "Improving risk evaluation in FMEA with cloud model and hierarchical TOPSIS method," IEEE Transactions on Fuzzy Systems, vol. 27, no. 1, pp. 84-95, 2018.

[27] E. K. Zavadskas, Z. Turskis, and S. Kildiene, "State of art surveys of overviews on MCDM/MADM methods," Technological and Economic Development of Economy, vol. 20, no. 1, pp. 165-179, 2014.

[28] M. Farajzadeh and A. Taghilo, "The wind energy potential zoning using GIS and fuzzy MCDM-based approach (study area: Zanjan province, Iran)," The International Journal of Humanities, vol. 20, no. 2, pp. 45-60, 2013.

[29] M. Rezaei-Shouroki, "The location optimization of wind turbine sites with using the MCDM approach: a case study," Energy Equipment and Systems, vol. 5, no. 2, pp. 165-187, 2017.

[30] G. Bos and N. Chatterjee, "Fuzzy hybrid MCDM approach for selection of wind turbine service technicians," Management Science Letters, vol. 6, no. 1, pp. 1-18, 2016.
[31] A. Beskese, A. Camci, G. T. Temur, and E. Erturk, "Wind turbine evaluation using the hesitant fuzzy AHP-TOPSIS method with a case in Turkey," Journal of Intelligent \& Fuzzy Systems, vol. 38, no. 1, pp. 997-1011, 2020.

[32] X. Deng and Y. Deng, "D-AHP method with different credibility of information," Soft Computing, vol. 23, no. 2, pp. 683-691, 2019.

[33] R. Bakhat and M. Rajaa, "Developing a novel grey integrated multi-criteria approach for enhancing the supplier selection procedure: a real-world case of textile company," Decision Science Letters, vol. 8, no. 3, pp. 211-224, 2019.

[34] N. Hemmati, M. R. Galankashi, D. M. Imani, and F. M. Rafiei, "An integrated fuzzy-AHP and TOPSIS approach for maintenance policy selection," International Journal of Quality \& Reliability Management, vol. 1, no. 1, pp. 1-24, 2019.

[35] M. Waris, S. Panigrahi, A. Mengal et al., "An application of analytic hierarchy process (AHP) for sustainable procurement of construction equipment: multicriteria-based decision framework for Malaysia," Mathematical Problems in Engineering, vol. 2019, Article ID 6391431, 20 pages, 2019.

[36] D. Pamučar and G. Ćirović, "The selection of transport and handling resources in logistics centers using multi-attributive border approximation area comparison (MABAC)," Expert Systems with Applications, vol. 42, no. 6, pp. 30163028, 2015.

[37] Y.-X. Xue, J.-X. You, X.-D. Lai, and H.-C. Liu, "An intervalvalued intuitionistic fuzzy MABAC approach for material selection with incomplete weight information," Applied Soft Computing, vol. 38, pp. 703-713, 2016.

[38] A. Debnath, J. Roy, S. Kar, E. Zavadskas, and J. Antucheviciene, "A hybrid MCDM approach for strategic project portfolio selection of agro by-products," Sustainability, vol. 9, no. 8, p. 1302, 2017.

[39] L. Gigović, D. Pamučar, D. Božanić, and S. Ljubojević, "Application of the GIS-DANP-MABAC multi-criteria model for selecting the location of wind farms: a case study of Vojvodina, Serbia," Renewable Energy, vol. 103, pp. 501-521, 2017.

[40] L. De Boer, E. Labro, and P. Morlacchi, "A review of methods supporting supplier selection," European Journal of Purchasing \& Supply Management, vol. 7, no. 2, pp. 75-89, 2001.

[41] D. Julong, "Introduction to grey system theory," The Journal of Grey System, vol. 1, no. 1, pp. 1-24, 1989.

[42] S. Liu, L. Tao, N. Xie, and Y. Yang, "On the new model system and framework of grey system theory," in Proceedings of the 2015 IEEE International Conference on Grey Systems and Intelligent Services (GSIS), pp. 1-11, IEEE, Leicester, UK, August 2015.

[43] H.-C. Liu, J.-X. You, X.-J. Fan, and Q.-L. Lin, "Failure mode and effects analysis using D numbers and grey relational projection method," Expert Systems with Applications, vol. 41, no. 10, pp. 4670-4679, 2014.

[44] N. Pancholi and M. G. Bhatt, "Multicriteria FMECA based decision-making for aluminium wire process rolling mill through COPRAS-G," Journal of Quality and Reliability Engineering, vol. 2016, Article ID 8421916, 8 pages, 2016.

[45] M.-L. Tseng, "A causal and effect decision making model of service quality expectation using grey-fuzzy DEMATEL approach," Expert Systems with Applications, vol. 36, no. 4, pp. 7738-7748, 2009.

[46] C. Bai and J. Sarkis, "A grey-based DEMATEL model for evaluating business process management critical success factors," International Journal of Production Economics, vol. 146, no. 1, pp. 281-292, 2013. 
[47] X. Xia, K. Govindan, and Q. Zhu, “Analyzing internal barriers for automotive parts remanufacturers in China using greyDEMATEL approach," Journal of Cleaner Production, vol. 87, pp. 811-825, 2015.

[48] M. Mendonça Silva, T. Poleto, L. Camara e Silva, A. P. Henriques de Gusmao, and A. P. Cabral Seixas Costa, "A grey theory based approach to big data risk management using FMEA," Mathematical Problems in Engineering, vol. 2016, Article ID 9175418, 15 pages, 2016.

[49] Q. Zhou and V. V. Thai, "Fuzzy and grey theories in failure mode and effect analysis for tanker equipment failure prediction," Safety Science, vol. 83, pp. 74-79, 2016.

[50] M. Omidvar and F. Nirumand, "Risk assessment using FMEA method and on the basis of MCDM, fuzzy logic and grey theory: a case study of overhead cranes," Health and Safety at Work, vol. 7, no. 1, pp. 63-76, 2017.

[51] G. M. Duman, E. Kongar, and S. M. Gupta, "A holistic greyMCDM approach for green supplier elicitation in responsible manufacturing," in Responsible Manufacturing, pp. 105-118, CRC Press, Boca Raton, FL, USA, 2019.

[52] S. Soua, P. Van Lieshout, A. Perera, T.-H. Gan, and B. Bridge, "Determination of the combined vibrational and acoustic emission signature of a wind turbine gearbox and generator shaft in service as a pre-requisite for effective condition monitoring," Renewable Energy, vol. 51, pp. 175-181, 2013.

[53] D. Milborrow, "Operation and maintenance costs compared and revealed," Wind Statistics, vol. 19, no. 3, p. 3, 2006.

[54] W. T. Chong, K. C. Pan, S. C. Poh et al., "Performance investigation of a power augmented vertical axis wind turbine for urban high-rise application," Renewable Energy, vol. 51, pp. 388-397, 2013.

[55] Y. Sinha and J. A. Steel, "A progressive study into offshore wind farm maintenance optimisation using risk based failure analysis," Renewable and Sustainable Energy Reviews, vol. 42, pp. 735-742, 2015.

[56] W. Y. Liu, B. P. Tang, J. G. Han, X. N. Lu, N. N. Hu, and Z. Z. He, "The structure healthy condition monitoring and fault diagnosis methods in wind turbines: a review," $R e$ newable and Sustainable Energy Reviews, vol. 44, pp. 466-472, 2015.

[57] D. Julong, "Introduction to grey system theory," The Journal of grey system, vol. 1, pp. 1-24, 1989.

[58] A. Vafadarnikjoo, M. Mobin, C. Salmon, and N. Javadian, “An integrated gray-fuzzy cause and effect approach to determine the most significant categories of project risks," in Proceedings of the IIE Annual Conference, p. 987, Institute of Industrial and Systems Engineers (IISE), Nashville, TN, USA, May 2015.

[59] S. Opricovic and G.-H. Tzeng, "Defuzzification within a multicriteria decision model," International Journal of Uncertainty, Fuzziness and Knowledge-Based Systems, vol. 11, no. 5, pp. 635-652, 2003.

[60] Y. Dou, Q. Zhu, and J. Sarkis, "Evaluating green supplier development programs with a grey-analytical network process-based methodology," European Journal of Operational Research, vol. 233, no. 2, pp. 420-431, 2014.

[61] A. Ulutas, N. Shukla, S. Kiridena, and P. Gibson, "A utilitydriven approach to supplier evaluation and selection: empirical validation of an integrated solution framework," International Journal of Production Research, vol. 54, no. 5, pp. 1554-1567, 2016.

[62] T. L. Saaty, "How to make a decision: the analytic hierarchy process," European Journal of Operational Research, vol. 48, no. 1, pp. 9-26, 1990.
[63] J. Benesty, J. Chen, Y. Huang, and I. Cohen, "Pearson correlation coefficient," in Noise Reduction in Speech Processing, pp. 1-4, Springer, Berlin, Heidelberg, 2009.

[64] H. S. Tooranloo and A. sadat Ayatollah, "A model for failure mode and effects analysis based on intuitionistic fuzzy approach," Applied Soft Computing, vol. 49, pp. 238-247, 2016. 